\title{
The highly attenuated oncolytic recombinant vaccinia virus GLV-1h68: comparative genomic features and the contribution of $F 14.5 L$ inactivation
}

\author{
Qian Zhang • Chunguang Liang • Yong A. Yu • \\ Nanhai Chen · Thomas Dandekar • Aladar A. Szalay
}

Received: 9 May 2009 / Accepted: 31 July 2009 / Published online: 22 August 2009

(C) The Author(s) 2009. This article is published with open access at Springerlink.com

\begin{abstract}
As a new anticancer treatment option, vaccinia virus (VACV) has shown remarkable antitumor activities (oncolysis) in preclinical studies, but potential infection of other organs remains a safety concern. We present here genome comparisons between the de novo sequence of GLV$1 \mathrm{~h} 68$, a recombinant VACV, and other VACVs. The identified differences in open reading frames (ORFs) include genes
\end{abstract}

Communicated by S. Hohmann.

Q. Zhang and C. Liang contributed equally.

Electronic supplementary material The online version of this article (doi:10.1007/s00438-009-0475-1) contains supplementary material, which is available to authorized users.

Q. Zhang · Y. A. Yu · N. Chen · A. A. Szalay $(\bowtie)$

Genelux Corporation, San Diego Science Center,

3030 Bunker Hill St., Ste. 310, San Diego, CA 92109, USA

e-mail: aaszalay@genelux.com

C. Liang $\cdot$ T. Dandekar $(\bowtie)$

Department of Bioinformatics, Biocenter,

University of Würzburg, Am Hubland,

97074 Würzburg, Germany

e-mail: dandekar@biozentrum.uni-wuerzburg.de

T. Dandekar

Structural and Computational Biology,

EMBL, Heidelberg, Germany

\section{A. A. Szalay}

Rudolf Virchow Center for Experimental Biomedicine, Institute for Biochemistry and Institute for Molecular Infection Biology, University of Würzburg, Am Hubland, 97074 Würzburg, Germany

\section{A. A. Szalay}

Radiation Oncology Department, Moores Cancer Center, University of California, San Diego, 3855 Health Sciences Drive \# 0843, La Jolla, CA 92093-0843, USA encoding host-range selection, virulence and immune modulation proteins, e.g., ankyrin-like proteins, serine proteinase inhibitor SPI-2/CrmA, tumor necrosis factor (TNF) receptor homolog CrmC, semaphorin-like and interleukin-1 receptor homolog proteins. Phylogenetic analyses indicate that GLV$1 \mathrm{~h} 68$ is closest to Lister strains but has lost several ORFs present in its parental LIVP strain, including genes encoding $\mathrm{CrmE}$ and a viral Golgi anti-apoptotic protein, v-GAAP. The reduced pathogenicity of GLV-1h68 is confirmed in male mice bearing $\mathrm{C} 6$ rat glioma and in immunocompetent mice bearing B16-F10 murine melanoma. The contribution of foreign gene expression cassettes in the F14.5L, J2R and A56R loci is analyzed, in particular the contribution of $F 14.5 \mathrm{~L}$ inactivation to the reduced virulence is demonstrated by comparing the virulence of GLV-1h68 with its $F 14.5 \mathrm{~L}$-null and revertant viruses. GLV-1h68 is a promising engineered VACV variant for anticancer therapy with tumor-specific replication, reduced pathogenicity and benign tissue tropism.

Keywords Virulence $\cdot$ Immune modulation - Tissue tropism $\cdot$ Foreign expression cassette $\cdot$ Phylogeny

$\begin{array}{ll}\text { Abbreviations } \\ \text { VACV } & \text { Vaccinia virus } \\ \text { CPV } & \text { Cowpox virus } \\ \text { OPV } & \text { Orthopoxvirus } \\ \text { GLV-1h68 } & \begin{array}{l}\text { Vaccinia virus strain GLV-1h68 (EU410304) } \\ \text { WR }\end{array} \\ & \text { Vaccinia virus strain Western } \\ \text { Reserve (AY243312) } \\ \text { COP } & \text { Vaccinia virus strain Copenhagen (M35027) } \\ \text { LIST } & \text { Vaccinia Lister major strain (AY678276) } \\ \text { LC } & \text { Vaccinia Lister isolate } \\ \text { VACV107 } & \begin{array}{l}\text { LC16mO (AY678277) } \\ \text { Vaccinia Lister } \\ \text { clone VACV107(DQ121394) }\end{array}\end{array}$




\begin{tabular}{|c|c|}
\hline ACAM & $\begin{array}{l}\text { Vaccinia virus strain } \\
\text { ACAM2000 (AY313847) }\end{array}$ \\
\hline DUKE & Vaccinia virus strain DUKE (DQ439815) \\
\hline MVA & Vaccinia virus strain Ankara (U94848) \\
\hline MP_ZAI & $\begin{array}{l}\text { Monkeypox virus strain } \\
\text { Zaire-96-I-16 (AF380138) }\end{array}$ \\
\hline MP_SL & $\begin{array}{l}\text { Monkeypox virus isolate } \\
\text { Sierra Leone (AY741551) }\end{array}$ \\
\hline CP_GRI & Cowpox virus strain GRI-90 (X94355) \\
\hline CAM_CMS & Camelpox virus CMS (AY009089) \\
\hline ECT_MOS & Ectromelia virus strain Moscow (AF012825) \\
\hline VAR_IND & Variola virus India-1967 (X69198) \\
\hline VAR_GAR & Variola virus Garcia-1966 (Y16780) \\
\hline VAR_BSH & Variola virus Bangladesh-1975 (L22578) \\
\hline
\end{tabular}

\section{Introduction}

Vaccinia virus (VACV) is the prototype virus of the orthopoxvirus genus in the poxvirus family. It has a linear, approximately 190-kb, double-stranded DNA genome, encoding more than 200 ORFs. VACV strains have been used extensively as vaccines and have played a central role in the eradication of the smallpox disease. More recently, research has focused on the potential of using VACV as an oncolytic virus for therapy of tumors. Many features associated with VACV are considered valuable for viral therapy, including the large cloning capacity, the natural tumor colonization capability, a short life cycle with strong lytic ability, and the capability to infect and replicate in human cells without causing natural disease in humans (Shen and Nemunaitis 2005). Several VACV vectors have shown remarkable antitumor and antimetastases results in preclinical studies; however, the significant level of infection in other organs remains a safety concern for systemic administration (Thorne et al. 2005). Our oncolytic VACV GLV-1h68 may be a potential improvement. To this end, its attenuation basis is studied here first by triple genome comparison, overall tissue tropism and key effects of engineered mutations.

VACV strains exhibit variations in virulence, as well as variations in host and tissue specificity or tissue tropism. Sequence analysis of VACV genomes has improved our understanding of the potential functions of viral gene products and host-virus interactions (Goebel et al. 1990; Antoine et al. 1998; Upton et al. 2003; Li et al. 2006). It is also known that several nonessential genes, such as $J 2 R$ (thymidine kinase TK) (Buller et al. 1985), C11R (secreted epidermal growth factor-like) (Buller et al. 1988), $A 56 R$ (hemagglutinin HA) (Shida et al. 1988), and B8R (soluble interferon-gamma receptor-like) (Verardi et al. 2001) result in reduced virulence when deleted or disrupted experimentally.

LIVP is a VACV vaccine strain originated from the Lister strain, which was adapted to calf skin in the Institute of Viral Preparations, Moscow, Russia (Al'tshtein et al. 1985). Western Reserve (WR) is derived from the New York City Board of Health (NYCBH) strain by repeated passages in the mouse brain (Henderson and Moss 1999). In a previous study, we reported that rVV-ruc-gfp, a recombinant LIVP carrying a fusion gene encoding light-emitting proteins, enters, replicates in, and reveals the locations of tumors in mice (Yu et al. 2004). Subsequently, a new recombinant virus, GLV-1h68, was constructed by inserting three expression cassettes into the $F 14.5 \mathrm{~L}, J 2 R$, and $A 56 R$ loci of the viral genome (Zhang et al. 2007). When administered intravenously, GLV-1h68 demonstrated remarkable tumor targeting and a much improved safety profile, as compared to its parental LIVP strains and the WR strains. In female nude mice bearing human GI-101A breast tumor xenografts that were injected with wt-WR or $\mathrm{TK}^{-} \mathrm{WR}$, a significantly elevated viral distribution was found in all organs examined, particularly in brain and ovarian tissues. In contrast, both the brain and ovaries were free of virus particles in mice injected with GLV-1h68.

To help understand the differences in the biodistribution between the LIVP and WR strains and the factors contributed to reduced virulence of GLV-1h68, we sequenced the genomic DNAs of wt-LIVP and GLV-1h68. We present here extensive genome comparisons between GLV-1h68, Lister, WR and Copenhagen (COP), based on the de novo sequence of GLV-1h68 and the functional analysis of individual ORFs. Since the LIVP strains exhibited significantly different tissue colonization patterns, as well as remarkably host-range restrictions compared to the neurovirulent WR strains (Zhang et al. 2007), we focus our analyses on genes suggested to be involved in tissue tropism, host-range restriction and immune modulation. A number of gene differences identified in these genomes support our observation of viral attenuation in GLV-1h68. The reduced pathogenicity of GLV-1h68, while remaining replication competent in tumors, was further confirmed in more tumor models, and the contribution of inactivated $F 14.5 \mathrm{~L}$ to the attenuation of GLV-1h68 was investigated by comparing the virulence of GLV-1h68 with its $F 14.5 \mathrm{~L}$-null and revertant viruses.

\section{Materials and methods}

Cells and virus strains

CV-1 African green monkey kidney fibroblast, C6 rat glioma, and B16-F10 murine melanoma cells were purchased from the American Type Culture Collection (ATCC, Manassas, VA). These cells were cultured in Dulbecco's modified Eagle's medium (DMEM), supplemented with antibiotic-antimycotic solution (penicillin $\mathrm{G} 100 \mathrm{U} \mathrm{ml}^{-1}$, 
amphotericin B $250 \mathrm{ng} \mathrm{ml}^{-1}$, streptomycin $100 \mathrm{U} \mathrm{ml}^{-1}$ ) and $10 \%$ fetal bovine serum (FBS) at $37^{\circ} \mathrm{C}$ under $5 \% \mathrm{CO}_{2}$. Mouse embryonic fibroblasts MEF (CF-1) were originally obtained from ATCC and cultured in DMEM with $15 \%$ FBS. The construction of recombinant VACV GLV-1h68 has been described previously (Zhang et al. 2007). Briefly, a Renilla luciferase-Aequorea green fluorescent protein $(R U C-G F P)$ fusion cDNA under the control of the vaccinia synthetic early/late promoter was inserted into the $F 14.5 \mathrm{~L}$ locus of wt-LIVP. One green plaque was clonally purified and designated as the single-mutant virus GLV-1d27. Then, a $\beta$-galactosidase (lac $Z$ ) encoding cDNA, under the control of p7.5 promoter, was inserted into the $T K$ locus of GLV$1 \mathrm{~d} 27$ to generate the double-mutant virus GLV-1f65, and a $\beta$-glucuronidase (gusA) encoding cDNA under the control of p11 promoter was inserted into the HA locus of GLV$1 \mathrm{f} 65$ to generate the triple-mutant virus GLV-1h68. All the recombinant viruses were purified through several rounds of plaque purification. Other strains included for comparison were WR wt and WR $T K^{-}$strain GLV-0b05, in which the $T K$ locus was disrupted by the insertion of RUC-GFP and lac $Z$ expression cassettes, as described previously (Zhang et al. 2007). The WR viruses were chosen because WR is the most extensively studied laboratory strain, and its wt genome sequence is readily available for comparative analysis.

\section{Purification of viral DNA for sequencing}

Both GLV-1h68 and wt-LIVP were propagated and titrated in CV-1 cell monolayers. CV-1 cells infected with GLV$1 \mathrm{~h} 68$ or wt-LIVP were harvested by centrifugation and disrupted by three cycles of freeze and thaw. Cell debris and nuclei were removed from cell lysates by low-speed centrifugation. The recovered viral particles were then purified by centrifugation through sucrose cushions and sucrose gradients (20-40\%) using established protocols (Joklik 1962). Genomic viral DNA was extracted from purified virions after treatment with proteinase $\mathrm{K}$ and followed by phenol-chloroform extraction, as described previously (Earl et al. 1998). The purified GLV-1h68 and wt-LIVP DNAs were used for sequencing by AGOWA GmbH (Germany).

\section{Sequence analysis of GLV-1h68}

The genomic DNA prepared from purified GLV-1h68 was sequenced by a shotgun approach and assembled by AGOWA GmbH. The pUC19 hybrid plasmids were sequenced to an $8 \times$ coverage, and only high quality reads (PHRED quality score: 20 ) were put into the assembly. Gap closure was performed by additional sequencing runs on the shotgun clones or by PCR using the viral genomic
DNA. Because the terminal hairpin loops were not sequenced, the leftmost nucleotide of the assembled sequences was arbitrarily designated as base number 1 . ORFs were predicted using multiple sequence analysis tools, including ORF Finder (http://www.ncbi.nih.gov/gorf/ gorf.html), GeneMark (Besemer and Borodovsky 2005) and Glimmer3 (Delcher et al. 2007), to enhance sensitivity and accuracy. ORFs that were predicted to be shorter than 50 amino acids were analyzed with BLAST further on other known virus genomes and recorded only when at least one highly identical hit with low $E$-value (below $10^{-6}$ ) was present.

The recombination regions in GLV-1h68, including $F 14.5 L, J 2 R, A 56 R$, were first replaced by the consensus sequence of corresponding regions of wt-LIVP. Afterward, the sequence was compared with the sequence of the WR strain using Smith-Waterman algorithm (Smith and Waterman 1981) on a Genematcher2 system. Bioperl modules (Stajich et al. 2002) were used to parse the reports automatically. The inGeno software was applied to analyze the rearrangement events (Liang and Dandekar 2006). The same comparison procedures described above were repeated on various poxvirus genomes. In addition to WR, we examined COP, MVA and Tiantan strains, and then the cowpox, camelpox, monkeypox and variola viruses. Subsequently, the annotation was improved and confirmed with Prosite (Sigrist et al. 2002) and PFAM/HMMER search (Bateman et al. 2004). The detailed motif hits are available in the supplementary list S1 (online at http://vaccinia.bioapps.biozentrum.uni-wuerzburg.de. The sequence data of GLV-1h68 has been submitted to NCBI database under GenBank accession no. EU410304).

Phylogenetic analysis

Phylogenetic analysis of GLV-1h68 was carried out on a conserved 82-kb nucleotide sequence, which is present in the central conserved region of 16 orthopoxvirus genomes (suppl. list S2). Corresponding regions were located from dot plots using the Dotter software (Sonnhammer and Durbin 1995) and aligned using MAFFT (Katoh et al. 2005). ClustalW was used to construct a Neighbor-Joining tree (Thompson et al. 1994), and its robustness was detected using bootstrap methods. NJPlot (Perrière and Gouy 1996) and SplitsTree4 (Huson and Bryant 2006) were used to analyze and plot the phylogenetic tree with bootstrapping.

\section{Detection of $c r m E$ gene by PCR}

The genomic DNAs from wt-LIVP, GLV-1d27, and GLV$1 \mathrm{~h} 68$ were used as the templates for PCR amplification of the specific $c r m E$ gene fragment. The primer sequences for 
crmE were 5'-GTCCGAGTGACACATTCACG-3' and 5'-ACCCACGACCACATTTTGTT-3', which correspond to the crmE gene (mO259R) 181,487-181,506 and 181,677-181,696, respectively, in Lister isolate LC16mO (Genbank accession no. AY678277). The primer sequences for the conserved F13L gene fragment, which served as the positive control, were 5'-CGCGTCATTTACTGGAGGAT-3' and 5'-GCAGTGCTAACTGGCAAACA-3', which correspond to 40,770-40,789 and 40,613-40,632, respectively, in the GLV-1h68 genome sequence, or 44,171-44,190 and 44,014-44,033, respectively, in the LC16mO genome sequence.

Sequencing of the right terminal fragment of wt-LIVP

The genomic DNAs from wt-LIVP and GLV-1h68 were used as the templates for PCR with the primer 5'-GCT ATGATTAACTCCCACGATACTATGC-3', which corresponds to nucleotides 178,812-178,839 (mO257L) in LC16mO or 188,293-188,320 (GL272) in GLV-1h68, and the primer 5'-CCTTACGACGTTTACATCGACGAG-3', which corresponds to 184,655-185,678 (mORTRO2R) in LC16mO or 199,241-199,264 (GL285) in GLV-1h68. The PCR products were checked on $1 \%$ agarose gel, and the major PCR product for wt-LIVP was cloned into pCRBlunt II-TOPO vector (Invitrogen), and one of the clones was sequenced by primer-walking. This sequence was compared with the corresponding gene segments from GLV1h68, WR and other Lister strains.

Construction of the F14.5L-revertant virus

To make the $F 14.5 L$-revertant virus (i.e. GLV-1e135), the $R U C-G F P$ expression cassette in GLV-1h68 was removed and the $F 14.5 \mathrm{~L}$ locus was restored to its wild-type sequence. The wild-type sequence spanning the F14.5L region (including $F 14.5 \mathrm{~L}$ and its flanking region, approximately $1.1 \mathrm{~kb}$ in length) of the wt LIVP genome was PCR amplified with primers 5'-ACTAGT (SpeI) TTCCCTCGT TCATCTAGCAAAAC- $3^{\prime}$ and 5'-AAGCTT (HindIII) TGAGGAGTATTGCGGGGCTA-3'. The PCR product was cloned into pCR-Blunt II-TOPO vector (Invitrogen) and the sequence of wt F14.5L was confirmed. The wt F14.5L was released by HindIII and SpeI, then subcloned into the pCR2.1 vector (Invitrogen) with the same cuts, in which a xanthine-guanine phosphoribosyltransferase (gpt) expression cassette, PCR amplified from pUCP7.5-gpt-1 (kindly provided by Dr. Kangla Tsung, University of California, San Francisco, USA), was inserted in the EcoRI site. The final construct pCR-wtF14.5L-gpt1 was confirmed by sequencing. This construct was used to make the recombinant virus GLV-1e135 from the parental virus, GLV-1h68, using the methods described by Falkner and Moss (1990).
Construction of the $F 14.5 L$-null virus

To generate a $F 14.5 L$-null virus, the $R U C-G F P$ expression cassette in the $F 14.5 \mathrm{~L}$ locus of GLV-1h68 was replaced by a short nonsense sequence, so that the $F 14.5 \mathrm{~L}$ would remain inactivated. This $F 14.5 L$-null virus, named GLV$1 \mathrm{~h} 71$, was engineered as follows. The left and right flanking sequences of F14.5L were PCR-amplified from wt LIVP using the primers $5^{\prime}$-GCGCATATGTAGAAGAATTGAT AAATATG-3' and 5'-GCCGCAGGATCCTGCGAAG CTTACAGACACGAATATGACTAAACCGATG-3' (left flank), 5'-GTCTGTAAGCTTCGCAGGATCCTGCGGCC GCCATCGTCGGTGTGTTGTC-3' and 5'-GCGGAATT CAGAGGATTACAACAAAAAGATG-3' (right flank). The two fragments were joined together using the method of gene-splicing by overlapping extension (Horton et al. 1993). The resulting fragment was digested with $N d e \mathrm{I}$ and EcoRI, and then cloned into the same-cut pUCP7.5-gpt-1 to yield pNCVVf14.51T. The recombinant virus GLV-1h71 was generated using pNCVVf14.51T from the parental virus GLV-1h68.

Viral replication in cell cultures

CV-1 $\left(1 \times 10^{5}\right)$, C6 $\left(2 \times 10^{5}\right)$, and B16-F10 $\left(2 \times 10^{5}\right)$ cells were seeded onto 24-well plates, and MEF (CF-1) $\left(1.1 \times 10^{5}\right)$ cells were seeded onto 12 -well plates. After $24 \mathrm{~h}$ in culture, the cells were infected with individual viruses at a multiplicity of infection (MOI) of 0.001 (for CV-1 or C6 cells) or an MOI of 0.01 (for MEF or B16-F10 cells). The cells were incubated at $37^{\circ} \mathrm{C}$ for $1 \mathrm{~h}$ with brief agitation every $10 \mathrm{~min}$ to allow infection to occur. The infection medium was removed and the cells were incubated in fresh growth medium until harvested at 24,48 , or $72 \mathrm{~h}$ after infection. Viral particles from the infected cells were released by a quick freeze-thaw cycle, and the titers determined as medium (pfu ml ${ }^{-1}$; plaque forming units per milliliter) in duplicate by plaque assay in CV-1 cell monolayers.

To investigate whether an inactivated $F 14.5 \mathrm{~L}$ affects the viral infection and replication in CV-1 cells, CV-1 cells seeded at $5 \times 10^{5}$ cells/well were infected with GLV-1h68, GLV-1h71 or GLV-1e135 at an MOI of 0.01 and harvested at 24,48 and $72 \mathrm{~h}$ after infection. The infection was performed in triplicate for each virus. Viral particles from infected cells were released and titrated as above, and the titers were determined as $\mathrm{pfu} / 10^{6}$ cells.

Viral distribution and pathogenicity studies

All mice were cared for and maintained in accordance with animal welfare regulations under an approved protocol by the Institutional Animal Care and Use Committee of LAB Research International Inc. and Explora Biolabs 
(San Diego, CA, USA). Mice with C6 xenograft tumors were developed by implanting C6 cells $\left(5 \times 10^{5}\right.$ cells in $100 \mu 1$ of PBS) subcutaneously into the right hind leg of 6- to 8-week-old male nude mice (NCI:Hsd:Athymic Nude-Foxn $1^{\text {nu }}$, Harlan). To compare the biodistribution and pathogenicity of individual virus strains, on day 7 after C6 cell implantation, when the median tumor volume was approximately $150 \mathrm{~mm}^{3}$, a single dose of virus $\left(1 \times 10^{7} \mathrm{pfu}\right.$ in $100 \mu \mathrm{l}$ PBS) was injected via the tail vein ( $n=4$ per virus). Body weight was monitored, and mice were checked daily for any sign of toxicity. Mice were sacrificed 14 days after virus injection, and the tumors and organs were analyzed for viral titers.

Similarly, the tissue distribution of viral particles and the pathogenicity of the viruses were also assessed in a syngeneic murine melanoma model. B16-F10 cells $\left(2 \times 10^{5}\right.$ cells $)$ in $40 \mu \mathrm{l}$ of PBS were injected into the foot pad of 6- to 8-week-old immunocompetent male C57BL/6 mice (Harlan). On day 18 after cell implantation, when the median tumor volume reached approximately $100 \mathrm{~mm}^{3}$, a single dose of virus $\left(1 \times 10^{7} \mathrm{pfu}\right.$ in $\left.100 \mu \mathrm{lBS}\right)$ was injected intravenously ( $n=4$ per virus). Mice were sacrificed 10 days after virus injection, and the tissue distribution of virus was analyzed.

To investigate whether a restored wt $F 14.5 \mathrm{~L}$ increased virulence in vivo, female nude mice (4-5 weeks old; Harlan; $n=8$ for PBS, GLV-1h68 or GLV-1h71 treated group, and $n=7$ for the GLV-1e135 group) were administrated an intranasal application of $2 \times 10^{6} \mathrm{pfu} / 20 \mu \mathrm{l}$ of individual virus per mouse. The mice were monitored twice weekly for body weight, and the survival rate was recorded for each treatment group during a period of 10 weeks.

\section{Statistical analysis}

Statistical analyses were performed with SPSS, version 11 (SPSS Inc., Chicago, IL, USA). Comparisons of treatment groups were made by analysis of variance (ANOVA), and the differences between the groups were analyzed with an LSD test when the ANOVA showed an overall significance. The survival rates for the different treatment groups were compared with the Log Rank test. Values of $p$ less than 0.05 were considered significant.

\section{Results}

Phylogenetic analysis

GLV-1h68 as a new virus with therapeutic potential was sequenced and then compared to other VACVs. We considered (see "Discussion") in particular the well known WR strain and the COP strain. Further phylogenetic analysis indicated that two Lister strains (Lister and LC16mO) were highly similar to GLV-1h68, and that all three strains had a recent common ancestor (Fig. 1). GLV-1h68 seemed to be closer to WR than to COP, which was also supported by sequence comparison results that indicated that GLV-1h68 shared more orthologous ORFs with WR than with COP (suppl. Fig. S1). Together with other VACV strains, such as ACAM, DUKE and MVA, these strains form a vaccinia cluster. The closest neighbors to the vaccinia cluster were cowpox virus (CP_GRI) and two monkeypox strains (MP_SL and MP_ZAI), followed by the camelpox strain (CAM_CMS). The three variola strains and the ectromelia virus strain (ECT_MOS) were relatively distinct in the generated tree.

\section{Genomic features of GLV-1h68}

The complete sequence of GLV-1h68 allowed bioinformatic analysis of all genes and genomic features detected. GLV$1 \mathrm{~h} 68$ was generated through three steps of construction as described previously. The resulting virus contained three foreign insertions of 1,958 bp, 8,606 bp, and 2,326 bp in the $F 14.5 L, J 2 R$, and A56R loci, respectively. With these three insertions, the size of the engineered GLV-1h68 virus was found to be 203,057 bp with $65.78 \% \mathrm{~A}+\mathrm{T}$ content. Without the insertions, the viral genome was found to be comprised of 190,167 bp with $66.57 \% \mathrm{~A}+\mathrm{T}$ content, and can be translated into 238 "major" ORFs (not embedded ORFs, see below). Most of the ORFs encoded protein products larger than 50 amino acids and were non-overlapping. Only a few weakly overlapped with other ORFs. In addition, 13 short ORFs were included as "major" ORFs since they are present in annotated ORFs of WR or COP. We also found 61 "minor" ORFs embedded in larger ORFs within the genome. In total, 299 potential ORFs, listed consecutively from left to right as they appeared in the genome, are summarized in suppl. Table S1 and S2. The homologs present in the WR genome are also listed for comparison, and the nomenclature for the COP genome is used as reference. The lengths, annotations and descriptions of some extremely short ORFs are listed in suppl. Table S3. The most striking differences among the ORFs of the three VACV strains are presented in suppl. Table S4. The numbers of vaccinia major and minor ORFs are summarized in suppl. Table S5.

Based on the annotation, we classified the major ORFs into six functional categories. Figure 2 shows their distribution in the viral genome. Sixty-one ORFs were likely to be involved in host interactions and immune modulation, and most of these were found to be positioned in the left and right terminal regions and the edges of the central conserved region. Fortynine ORFs encoding proteins involved in viral structure and assembly were located in the central conserved region, including viral core proteins, plaque forming, and EEV and IEV 
Fig. 1 Phylogenetic tree constructed from orthopoxvirus genomes. The unrooted tree was generated by neighbour-joining, comparing highly conserved $82 \mathrm{~kb}$ nucleotide sequence among 16 OPV genomes. Bootstrapping values (percentage; 1,000 trials) are shown beside the nodes. Please see the list of abbreviations and accession numbers for virus identification

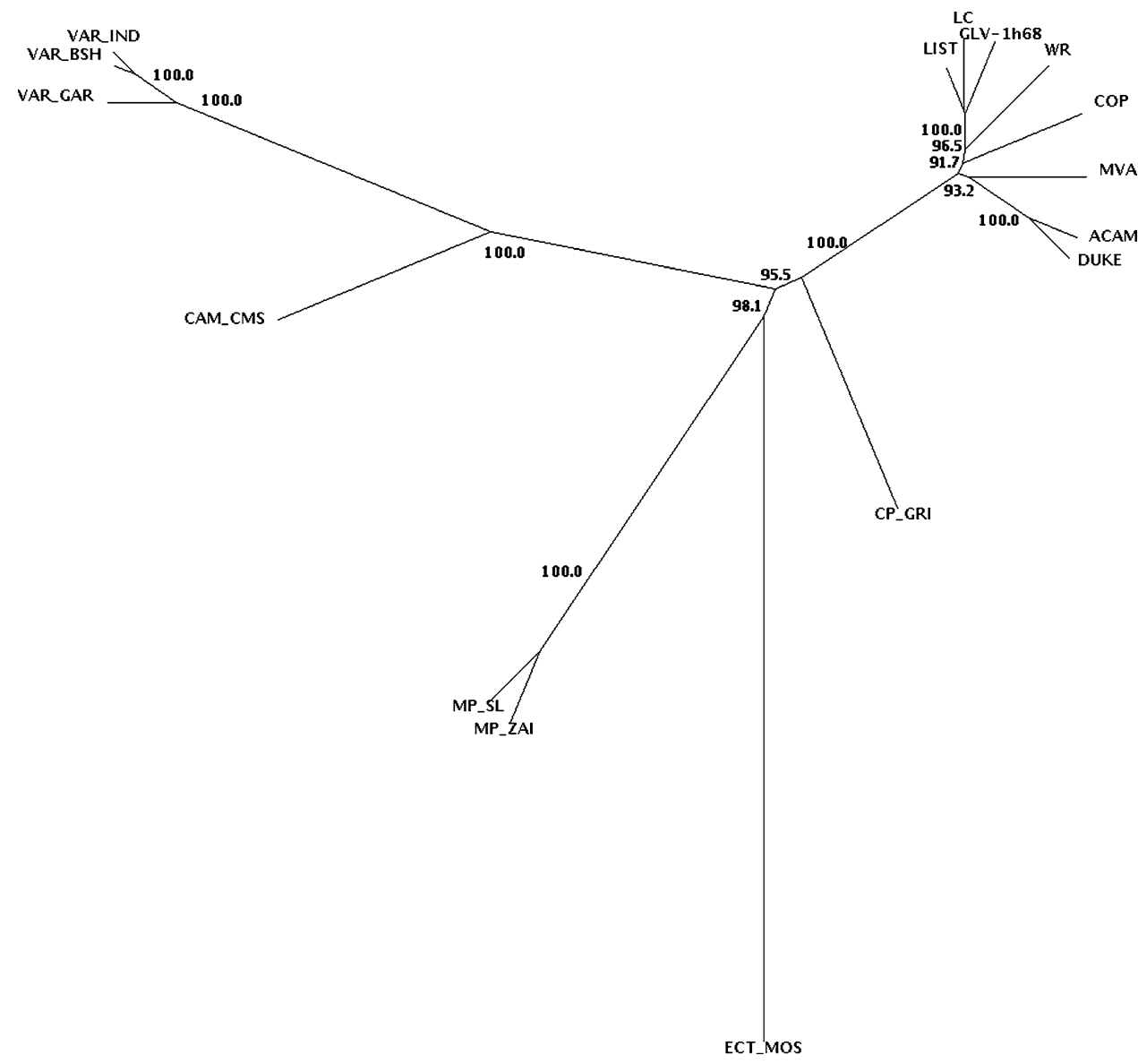

membrane proteins (pink). Genes involved in metabolism (41 ORFs, orange) and genes involved in DNA replication, RNA transcription and modifications (28 ORFs, green) were also located in the central conserved region. The functions of 58 ORFs remained uncertain or unidentified (marked with clear arrows). GL068 (F14.5L) was split into two parts by the $R U C-G F P$ expression cassette (marked in gray, Fig. 2). Other foreign insertions included lac $Z$ in the $T K$ locus, and gus $A$ in the $H A$ locus.

The sequence data for the wt-LIVP showed sub-strains and heterogeneity at the termini. There was a central conserved genomic region (from GL016 to GL276). It showed an unambiguous single nucleotide substitution (a GAA codon was changed into TAA as compared to the sequence in WR or COP) in the middle of the $J 2 R$ gene that created a premature stop codon. We further performed an in vitro assay (Mackett et al. 1982) which also demonstrated that the LIVP strain indeed had a TK negative phenotype for its wild type population (data not shown).

Comparison of ORFs involved in host-range selection and immune modulation

The majority of the differences in ORFs among the GLV1h68, WR and COP strains can be attributed to the ORFs encoding ankyrin-like proteins (Table 1). Table 1 presents additionally a variety of viral encoded complement, cytokine and chemokine inhibitors, and caspase inhibitors. Most of these immunomodulators were very well conserved among three VACV strains, usually with over $99 \%$ identity in the amino acid sequences. However, we found several genes that were different in GLV-1h68 than in WR. GLV-1h68 had an intact $A 39 R$, encoding a semaphorin-like protein, whereas, in WR, this protein was not expressed due to the presence of a frame-shift mutation. In addition, the TNF-receptor-like protein (vTNFR) encoded by $A 53 R$ gene seemed to be intact in GLV-1h68, but was fragmented in WR. On the other hand, SPI-2/CrmA, which inhibits Fasmediated apoptosis, was intact in WR (WR195) but fragmented in GLV-1h68. It is also interesting to note that GLV-1h68 had two copies of genes encoding for serine protease inhibitor (SPI-1) and interleukin-18-binding protein, whereas in WR only one copy of each was present.

\section{GLV-1h68 is a unique isolate from LIVP}

Specific genomic features point to GLV-1h68 being a unique isolate from LIVP. Thus VACV strains USSR, Lister, and Evans produce an additional soluble vTNFR, named CrmE. To our surprise, however, we could not 


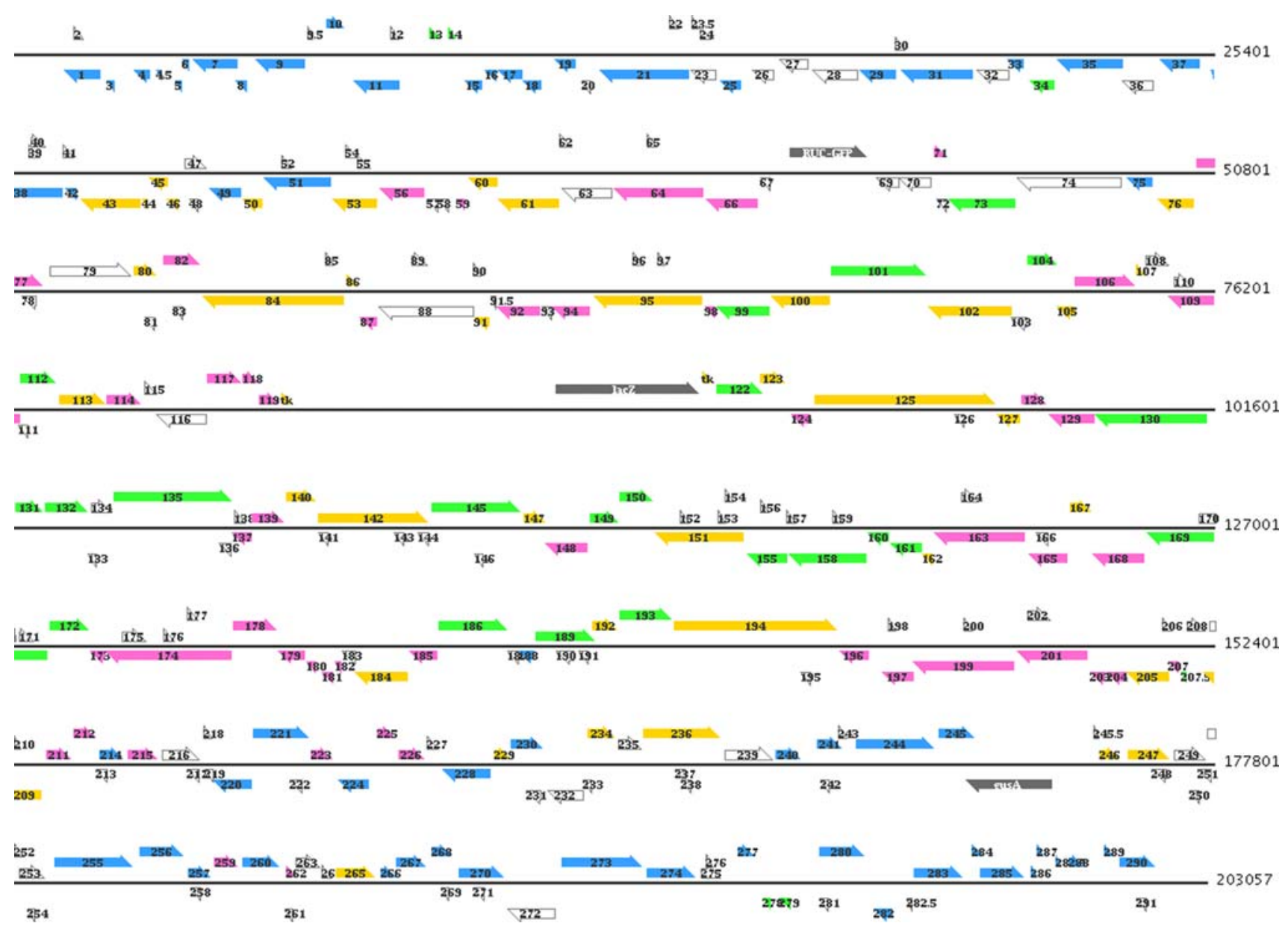

Fig. 2 GLV-1h68 genome map. Predicted ORFs and their lengths are represented by colored arrows. Sixty-one ORFs involved in host interactions and immune modulation are shown in blue. Located mostly in the central conserved region are genes involved in: viral structure and assembly (49 ORFs, shown in pink), metabolism (41 ORFs, shown in

orange) and DNA replication, RNA transcription and modifications (28 ORFs, in green). The functions of 58 ORFs remain uncertain (marked with clear arrows). The three foreign insertions are marked in dark grey. The genome size is indicated on the right (nucleotides)

identify a crmE-like sequence in the genome of GLV-1h68. To confirm this finding, the genomic DNAs from wt-LIVP, GLV-1d27, and GLV-1h68 viruses were used as the templates for PCR amplification of the specific $c r m E$ gene fragment. The PCR results indicated that wt-LIVP was $\mathrm{crmE}$ positive, but that GLV-1d27 and GLV-1h68 were not (Fig. 3a).

This unexpected finding regarding the loss of the $\mathrm{crmE}$ gene led us to further analyze the differences between GLV-1h68, wt-LIVP and other Lister strains. The genomic DNAs from GLV-1h68 and wt-LIVP were used as the templates for PCR with primers corresponding to $G L 272$ and GL285 in GLV-1h68 or $m O 257 L$ and $m O R T R 02 R$ in LC16mO, respectively. As predicted, the PCR product for GLV-1h68 was 10,971 bps (Fig. 3b), which was also the size of the PCR product from its parental virus GLV-1d27 or GLV-1f65 (data not shown). The PCR product for wt-LIVP, however, was much smaller, close to the size predicted for LC16mO (5,866 bps) (Fig. 3b).

The sequence of the major PCR product from wt-LIVP was analyzed and compared with the corresponding regions from GLV-1h68, WR, and other Lister strains (e.g. LC16mO) (Fig. 4). In addition to the missing $\mathrm{crmE}$ gene (ORF mO259R), mO260R, mO261R, mO262L and $m O R T R 01 R$ (present in the Lister strain LC16mO and wtLIVP) were also missing in GLV-1h68. $m O 261 R$ encodes a newly identified anti-apoptotic protein that is located in the Golgi, so-called viral Golgi anti-apoptotic protein (v-GAAP).

\section{GLV-1h68 carries genes of WR-like origin}

In contrast to the missing Lister genes, GL274 (encoding IFN- $\alpha / \beta$-receptor-like secreted glycoprotein) and GL275 (encoding ankyrin-like protein) were found in the GLV$1 \mathrm{~h} 68$ genome, with orthologs present in both WR and COP. These ORFs were absent from wt-LIVP, and all other Lister strains or derivatives sequenced (AY678275, 
Table 1 Major ORFs involved in host-range selection and immune modulation

\begin{tabular}{|c|c|c|c|c|c|c|c|c|c|c|c|c|c|}
\hline \multicolumn{7}{|c|}{ ORFs involved in host-range selection, including ankyrin-like proteins } & \multicolumn{7}{|c|}{ ORFs involved in modulation of host immune system } \\
\hline \multicolumn{3}{|c|}{ ORF locus_tag } & \multicolumn{3}{|c|}{ Translation } & \multirow{2}{*}{ Annotation } & \multicolumn{3}{|c|}{ ORF locus_tag } & \multicolumn{3}{|c|}{ Translation } & \multirow{2}{*}{ Annotation } \\
\hline GLV-1h68 & WR & COP & 1 h68 & WR & COP & & GLV-1h68 & WR & COP & $1 \mathrm{~h} 68$ & WR & COP & \\
\hline- & WR006|213 & C21L|B27R & - & 64 & 113 & Putative, ankyrin-like protein & GL001|290 & WR001/218 & C23L|B29R & 258 & 244 & 244 & Chemokine-binding protein \\
\hline- & WR007|212 & $\mathrm{C} 20 \mathrm{~L} \mid \mathrm{B} 26 \mathrm{R}$ & - & 109 & 103 & Putative, ankyrin-like protein & GL003|289* & & & 63 & 52 & 52 & Fragment of TNF receptor \\
\hline- & $\begin{array}{l}\text { WR008|211 } \\
*\end{array}$ & $\mathrm{C} 19 \mathrm{~L} \mid \mathrm{B} 25 \mathrm{R}$ & - & 112 & 259 & Putative, ankyrin-like protein & GL004|288 & WR004/215 & $\mathrm{C} 22 \mathrm{~L} \mid \mathrm{B} 28 \mathrm{R}$ & 122 & 122 & 122 & TNF- $\alpha$-receptor-like \\
\hline GL005|287 & - & - & 52 & - & - & Ankyrin-like protein & GL009|283 & WR205 & $\mathrm{C} 12 \mathrm{~L}$ & 354 & 353 & 353 & SPI-1 \\
\hline GL006|286* & - & $\mathrm{C} 18 \mathrm{~L} \mid \mathrm{B} 24 \mathrm{R}$ & 49 & - & 150 & Putative, host-range protein & GL010|282 & WR009/210 & C11R & 138 & 140 & 142 & VGF \\
\hline GL007|285* & - & $C 17 \mid \mathrm{B} 23 \mathrm{R}$ & 321 & - & 386 & Putative, host-range & GL011|280 & WR010/209 & $\mathrm{C} 10 \mathrm{~L}$ & 331 & 331 & 331 & IL-1 receptor antagonist \\
\hline GL008|284* & $1-$ & C17L|B23R & 86 & - & 386 & polypeptide & GL015|277 & WR013 & - & 124 & 126 & - & IL-18-binding protein \\
\hline GL016 & WRO14 & - & 90 & 237 & - & Ankyrin-like protein & GL029 & WR025 & C3L & 263 & 263 & 263 & Secreted complement binding \\
\hline GL017 & WR014 & - & 179 & 237 & - & Ankyrin-like protein & GL031 & WR026 & $\mathrm{C} 2 \mathrm{~L}$ & 512 & 512 & 512 & Kelch-like protein \\
\hline GL018 & WR015 & - & 137 & 137 & - & Ankyrin-like protein & GL033 & WR028 & N1L & 117 & 117 & 117 & $\begin{array}{l}\text { B cell lymphoma-2 (bcl-2)-like } \\
\text { protein }\end{array}$ \\
\hline \multirow{2}{*}{ GL019 } & WR016* & - & 157 & 77 & - & Ankyrin-like protein & GL038 & WR033 & K2L & 369 & 369 & 369 & SPI-like protein \\
\hline & WR017* & |- & 157 & 71 & - & Ankyrin-like protein & GLO42 & WR034 & K3L & 88 & 88 & 88 & Interferon resistance protein \\
\hline GL021 & WR019 & C9L & 634 & 634 & 634 & Ankyrin-like protein & GL051 & WR042 & F3L & 480 & 480 & 480 & Kelch-like protein \\
\hline GL025 & WR021 & C7L & 150 & 150 & 150 & Host-range protein & GL075 & WR059 & E3L & 190 & 190 & 190 & dsRNA binding protein \\
\hline GL035 & WR030 & M1L & 469 & 472 & 472 & Ankyrin-like protein & GL214 & WR158 & A35R & 176 & 176 & 176 & Virulence factor \\
\hline GL037 & WR032 & K1L & 134 & 134 & 136 & Ankyrin-like protein & GL220 & WR162 & A38L & 277 & 277 & 277 & CD47-like membrane protein \\
\hline GL075 & WR059 & E3L & 190 & 190 & 190 & dsRNA binding protein & \multirow{2}{*}{ GL221 } & WR163 & \multirow{2}{*}{$A 39 R$} & \multirow{2}{*}{403} & 295 & \multirow{2}{*}{403} & \multirow{2}{*}{ Semaphorin-like protein } \\
\hline GL255 & WR186 & B4R & 558 & 558 & 558 & Ankyrin-like protein & & WR164 & & & 142 & & \\
\hline GL256 & WR187 & B5R & 317 & 317 & 317 & EEV type-I memb. glycoprot. & GL224 & WR166 & A41L & 219 & 219 & 219 & Host defense modulator \\
\hline GL257 & WR188 & B6R & 173 & 173 & 173 & Ankyrin-like protein & GL228 & WR170 & A44L & 346 & 346 & 346 & Hydroxysteroid dehydrogenase \\
\hline \multirow[t]{11}{*}{ GL273 } & \multirow[t]{11}{*}{ WR199 } & \multirow[t]{11}{*}{ B18R } & \multirow[t]{11}{*}{574} & \multirow[t]{11}{*}{574} & \multirow[t]{11}{*}{574} & \multirow[t]{11}{*}{ Ankyrin-like protein } & GL230 & WR172 & A46R & 236 & 240 & 214 & \multirow{2}{*}{$\begin{array}{l}\text { IL-1 signaling inhibitor } \\
\text { Intracellular TLR and IL-1 } \\
\text { signaling inhibitor }\end{array}$} \\
\hline & & & & & & & GL240 & WR178 & $A 52 R$ & 190 & 190 & 190 & \\
\hline & & & & & & & GL241 & WR179 & A53R & 186 & 103 & 103 & TNF receptor \\
\hline & & & & & & & GL244 & WR180 & A55R & 564 & 564 & 564 & Kelch-like protein \\
\hline & & & & & & & GL245 & WR181 & A56R & 310 & 310 & 310 & Hemagglutinin \\
\hline & & & & & & & GL260 & WR190 & B8R & 272 & 272 & 272 & IFN- $\gamma$-like protein \\
\hline & & & & & & & GL266 & WR195 & B13R & 116 & 345 & 116 & SPI-2/CrmA, IL-1 convertase \\
\hline & & & & & & & GL267 & WR195 & B14R & 222 & 345 & 222 & SPI-2/CrmA, IL-1 convertase \\
\hline & & & & & & & GL268 & WR196 & B15R & 149 & 149 & 149 & IL-1 binding protein \\
\hline & & & & & & & GL270 & WR197 & B16R & 326 & 326 & 290 & IL-1-beta-inhibitor \\
\hline & & & & & & & GL274 & WR200 & B19R & 351 & 351 & 353 & IFN- $\alpha / \beta$-receptor-like \\
\hline
\end{tabular}

${ }^{a}$ Denotes the corresponding ORF regions present in both WR and COP strains, with locations listed in the suppl. Table S3

b Frame-shift mutation

- , No ortholog present

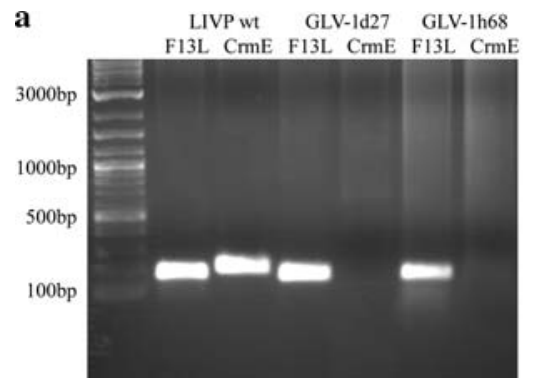

b

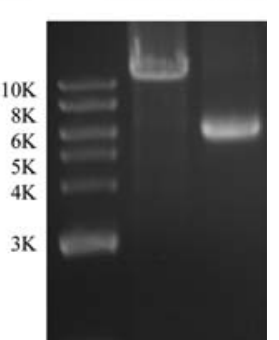

Fig. 3 The PCR results indicate that the parental strain of GLV-1h68 was a unique isolate from LIVP. a The genomic DNAs from wt-LIVP, GLV1d27, and GLV-1h68 viruses were used as the templates for PCR amplification of the specific $c r m E$ gene fragment and the conserved F13L gene fragment. The PCR results show that, while wt-LIVP population was $\mathrm{crmE}$ positive, GLV-1d27 and GLV-1h68 were not; $\mathbf{b}$ The genomic DNAs from wt-LIVP and GLV-1h68 were used as the templates for PCR with primers corresponding to $m O 257 L$ and $m O R T R 02 R$ in LC16mO or GL272 and GL285 in GLV-1h68, respectively. The PCR product for GLV-1h68 was $10,971 \mathrm{bps}$ as predicted. The major PCR product for wt-LIVP was much smaller, similar to the size predicted for $\mathrm{LC} 16 \mathrm{mO}(5,866 \mathrm{bps})$. This further confirms that GLV-1h68 was derived from a unique isolate of LIVP

AY678276, AY678277, DQ121394). Moreover, the neighboring ORF upstream of $\mathrm{crmE}$ in the Lister strain, $\mathrm{mO} 258 \mathrm{R}$ (413aa), was extended into GL273 (574aa) in GLV-1h68, suggesting an island of sequence insertion within and following this ORF. Considering the length differences of the inverted terminal repeat (ITR) between GLV-1h68 and other Lister strains, we decided to analyze the ORFs that were absent from the right terminus of the Lister strains, i.e., GL277-283. The comparison results indicated that these ORFs were copies from the left terminus, including $m O 008 R$ which were fragmented into GLO13/279 and GL014/278 (Fig. 4). This additional copy of nucleotide sequence largely extended the ITR size in GLV-1h68 in comparison to its parental LIVP or Lister strain.

Viral replication in different cell lines

In our previous studies (Zhang et al. 2007), GLV-1h68 was evaluated in human breast tumor cell line GI-101A to examine whether insertions in the $F 14.5 L, J 2 R$ or $A 56 R$ locus affected its activity. In this study, complementary to the broad comparison of genomic and pathogenomic features just detailed, infection studies were carried out in further tumor cell lines to compare the infectivity and replication of individual recombinant viruses (Fig. 5b). In CV-1 


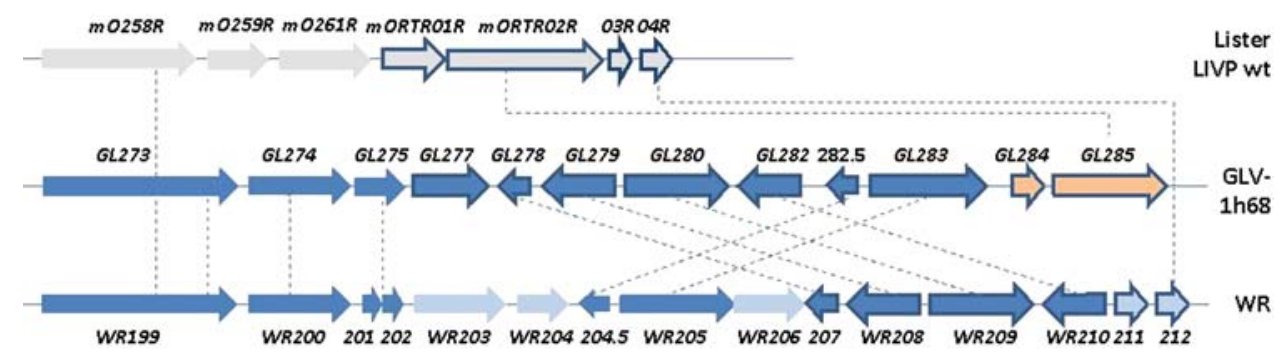

Fig. 4 GLV-1h68 showed a unique genetic makeup that was distinct from its parental LIVP strain. Genes outlined by navy blue have corresponding paralogs in the left terminus. Genes in light blue are unique for WR strain, and those in light orange are unique for GLV-1h68. The orthologous genes are linked by dashed lines. GL277 (encoding inter-

cells all recombinant viruses replicated very well, and showed comparable yield, similar to wt-WR and wt-LIVP viruses. In C6 glioma cells, however, the recombinant viruses, and particularly GLV-1h68, showed a reduced replication of up to $10^{2}$-fold, compared to wt-LIVP (Fig. 5b). Cells of mouse origins required a higher MOI for detectable infection, which was true for both primary MEFs and the tumor cell line B16-F10. At an MOI of 0.01, the wt-LIVP replicated fairly well in MEFs, but the three recombinant LIVP viruses did not replicate. In B16-F10 melanoma cells, all viruses showed limited infection and replication (Fig. 5b).

\section{Pathogenicity of individual VACVs}

The pathogenicity of individual VACVs was already evaluated in female nude mice bearing human GI-101A xenografts (Zhang et al. 2007). The current study examined whether the individual VACVs showed differential pathogenicity in male mice after a single intravenous injection of $1 \times 10^{7} \mathrm{pfu}$ of virus, and whether an intact immune system could overcome the infections of VACVs. In C6 gliomabearing nude male mice, wt-WR was extremely virulent (Fig. 5c). Mice exhibited over $30 \%$ body weight loss by day 7 after virus injection, and all mice died by day 11 . Pocks on the tail and footpad and swelling of the mouth region were observed. Male mice injected with the $\mathrm{TK}^{-}$ WR strain lost $22 \%$ of their body weight by the end of the 14 days, similar to mice injected with wt-LIVP strain. In contrast, no significant weight loss was observed in mice injected with GLV-1h68 or other recombinant viruses. The immunocompetent C57BL/6 mice, bearing B16-F10 melanoma on foot pad, showed a greater tolerance to viral pathogenicity (Fig. 5c). All mice injected with the wt-WR strain survived for the duration of the study; however, on average over $10 \%$ of body weight was lost by day 10 . Male mice injected with the $\mathrm{TK}^{-}$WR or the LIVP strains showed little weight loss, suggesting that a competent T-cell system plays an important role in fighting against virus infection. leukin-18-binding protein) in GLV-1h68 had an ortholog WRO13 in the left terminus of WR genome. The majority of the wt-LIVP population had sequences in the CrmE $(m O 259 R)$ and v-GAAP $(m O 261 R)$ gene segments that are similar to other Lister strains

Tissue distribution of individual VACVs

We next looked at virus localization in individual tissues. Table 2 summarizes the virus distribution data in C6 tumor-bearing nude male mice after intravenous injection of individual virus strains. In this experiment, mouse organs were collected 14 days after virus injection. However, mice injected with wt-WR were sacrificed and analyzed on day 7 due to extreme toxicity. As shown in Table 2, the wt-WR injection resulted in a broader distribution of the virus in healthy mouse tissues, including an alarmingly high titer in the brain. The inactivation of the $T K$ gene led to significant reductions in the viral titers in mice injected with the $T K^{-}$WR strain; however, viral titers of up to $10^{6} \mathrm{pfu}$ per gram of tissue were still present in the brain tissues. The wt-LIVP, on the other hand, showed a markedly different distribution pattern. No viral particles were recovered from the brain tissues in three of the four tumor-bearing mice. Mice injected with GLV-1h68 had no virus recovery from brain, testis, or bladder tissues, and very low levels of viral particles recovered from kidney, liver, or heart tissues in only one of the four mice. Viral particles were also recovered from the spleen tissues in two of the mice. Lung tissues seemed to be relatively susceptible to the GLV-1h68 infection, with up to $10^{3}$ pfu of the virus per gram of tissue found in three mice. The implanted C6 glioma tumors, however, were colonized heavily with either WR or LIVP strains. The greatly reduced biodistribution of GLV-1h68 in various mouse organs was in agreement with the viral replication data in mouse primary cells (MEFs in Fig. 5b).

In melanoma-bearing immunocompetent mice, no virus particles were recovered from non-tumor tissues 10 days after the virus injection (Table 2), even for mice injected with wt WR, suggesting the importance of $\mathrm{T}$ cells in the fight against viral infection. The viral titers were also lower in the tumor tissues, in parallel with the in vitro replication data (Fig. 5b). 
Fig. 5 Replication and pathogenicity of different VACV strains. a Genetic constructs for different VACV strains (reproduced from Zhang et al. 2007). A single base substitution created a premature stop codon; therefore, the TK gene in LIVP wt or GLV-1d27 was naturally inactivated. pSEL, VACV synthetic early late promoter; p7.5 and p11K, VACV 7.5 early/late and $11 \mathrm{k}$ promoters; TFR, human transferrin receptor. $\mathbf{b}$ The replication of different VACV strains was studied in various cell cultures. $\mathbf{c}$ The pathogenicity was evaluated by change in body weight after a single, intravenous injection of each individual virus at $10^{7} \mathrm{pfu}$ per mouse, in nude mice bearing C6 glioma, and C57BL/6 mice bearing B16-F10 melanoma. Change of body weight (\%) was calculated as follows:

$\left[\left(b^{\prime}-t^{\prime}\right)-(b-t)\right] \times 100 /$ $(b-t)$, where $b$ and $t$ are the body weight and tumor weight (estimated by tumor size; $1 \mathrm{~cm}^{3}=1 \mathrm{~g}$ ) on the day of virus injection, and $b^{\prime}$ and $t^{\prime}$ are the corresponding weights on the day of monitoring. The average weight change for each group is presented
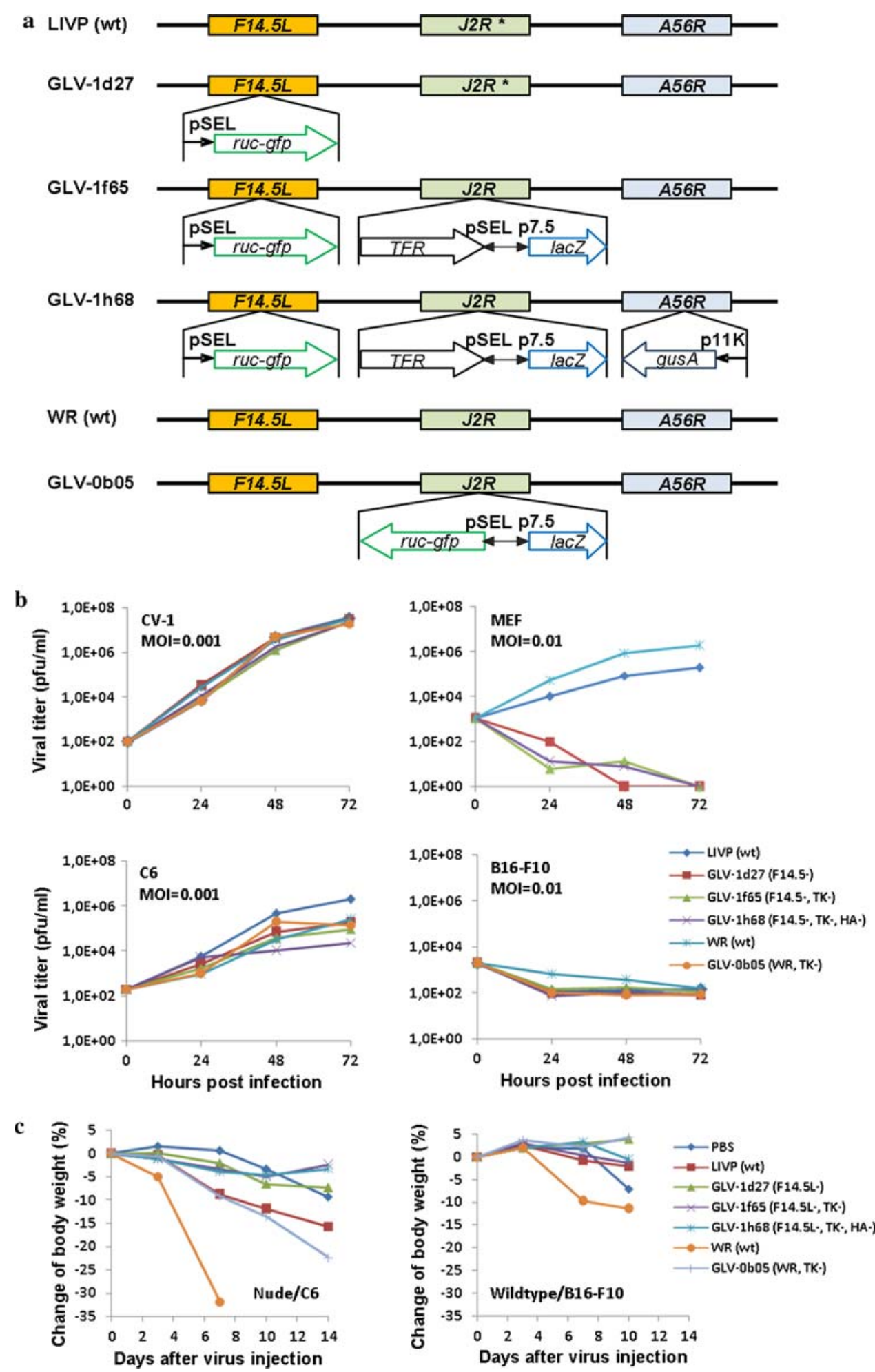

The contribution of $F 14.5 \mathrm{~L}$ inactivation

Given the promising data on GLV-1h68 from infection studies and genome comparisons, direct experimental tests probed next the pathogenicity effect of individual genes. Unlike the well known $T K$ or $H A$ gene in the VACV genome, the $F 14.5 \mathrm{~L}$ is relatively new and less studied. We therefore focused our direct experimental tests on whether the insertion of an expression cassette in the F14.5L contributed to the attenuation of GLV-1h68. We constructed an F14.5L-revertant virus GLV-1e135, in which the F14.5L was restored to its wild-type LIVP sequence (Fig. 6a). To 
Table 2 Distribution of VACV in healthy tissues versus tumors

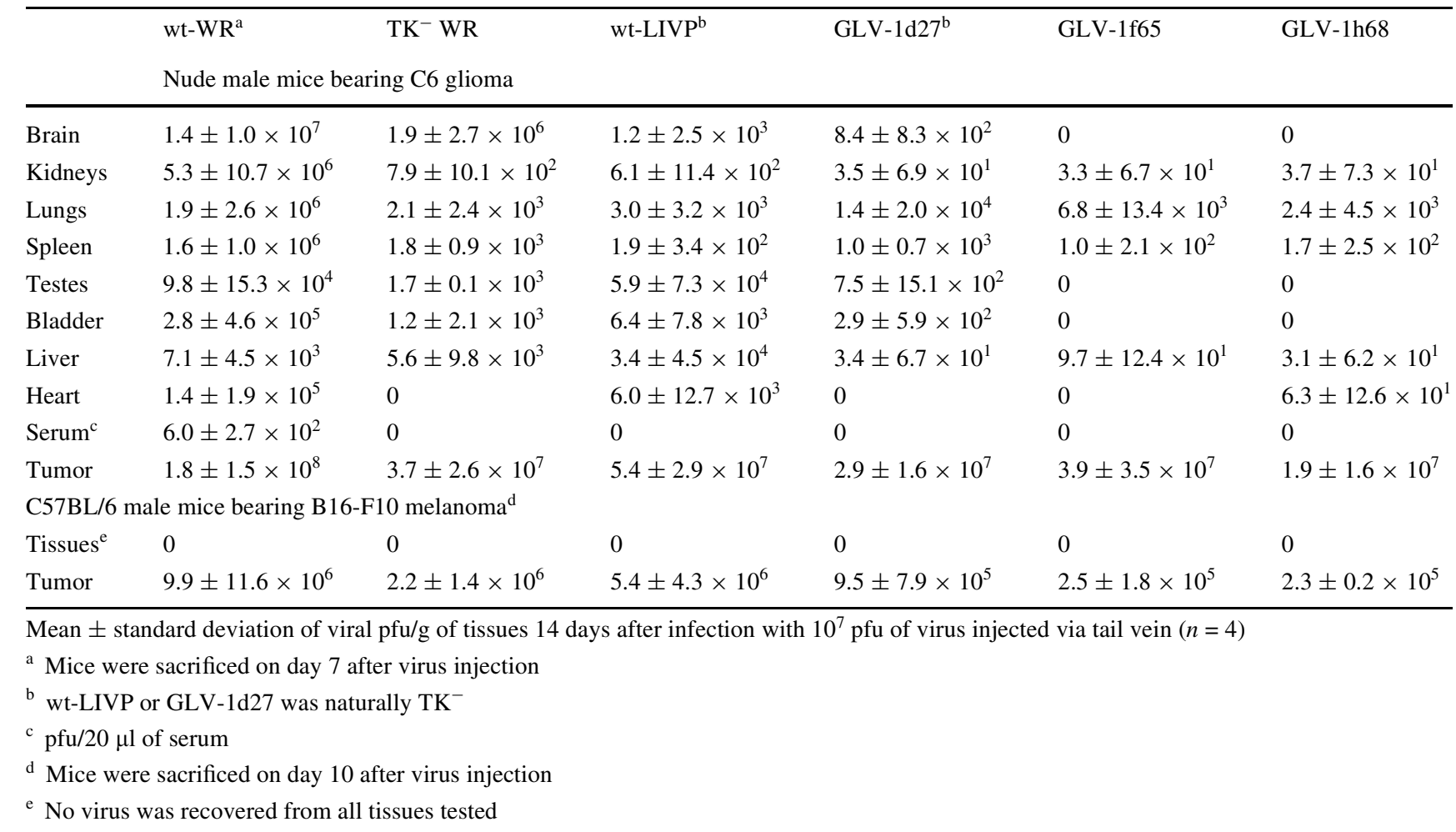

differentiate the contribution of the inactivation of $F 14.5 \mathrm{~L}$ by itself from the contribution by the RUC-GFP expression, GLV-1h71, an $F 14.5 L$-null virus, was used as a control in which a short nonsense sequence was inserted into the F14.5L locus to interrupt the $F 14.5 \mathrm{~L}$ gene (Fig. 6a). GLV1e135 was first studied in CV-1 cells, and its infection and/ or replication capacities were compared to GLV-1h68 or GLV-1h71. There appeared to be an increase in virus infection and/or replication for GLV-1e135 and GLV-1h71 compared to GLV-1h68 (Fig. 6b). At $24 \mathrm{~h}$ after the infection, the viral titer recovered from the infected CV-1 cells was the highest for GLV-1e135 $\left(6.9 \times 10^{6} \mathrm{pfu} / 10^{6}\right.$ cells, $p<0.001$ vs. GLV-1h71 or GLV-1h68), followed by GLV$1 \mathrm{~h} 71\left(2.5 \times 10^{6} \mathrm{pfu} / 10^{6}\right.$ cells $)$, which was also significantly $(p=0.002)$ higher than that of GLV-1h68 $\left(1.0 \times 10^{6} \mathrm{pfu} /\right.$ $10^{6}$ cells).

To study the virulence of the $F 14.5 \mathrm{~L}$-revertant and F14.5L-null viruses, nude female mice were administrated intranasally with $2 \times 10^{6} \mathrm{pfu} / 20 \mu \mathrm{l}$ of the individual viruses, including GLV-1h68, GLV-1e135, and GLV-1h71. The mice were monitored twice weekly for body weight, and the survival rate was recorded for each treatment group for a period of 10 weeks (Fig. 6c, d). Two of the mice treated with GLV-1h68 died during this study. The difference between the PBS treated group and the GLV-1h68treated group was not significant ( $p=0.143$, log rank test). Two more mice died in the GLV-1h71-treated group.
However, the survival rate for the GLV-1h71 group was not significantly different from that of the GLV-1h68 group $(p=0.361)$. In contrast, all mice in the GLV-1e135-treated group died during the study. This was a statistically significant difference from the GLV-1h68 treated group $(p=0.0069)$, even though the difference was not significant when compared to the GLV-1h71 group ( $p=0.068$ ).

In contrast to a typical acute toxicity study, the application dosage in this study was purposely kept low so that the subtle differences between recombinant viruses became obvious over the time. All mice died during the study had lost noticeable amounts of weight before their deaths (Fig. 6d). When the body weight for those surviving mice was compared at the end of the study, the ANOVA showed an overall significance of 0.001 . The mean body weight in the GLV-1h71-treated mice was $21.6 \mathrm{~g}$, which was significantly lower than that of the GLV-1h68-treated group (mean $=24.6 \mathrm{~g}, p=0.004$ ) or the PBS control mice (mean $=25.5 \mathrm{~g}, p<0.001$ ). The virulence of these recombinant viruses seemed to correlate well with their titers in the CV-1 cells $24 \mathrm{~h}$ after virus infections (Fig. 6b).

\section{Discussion}

In this study, we compared the pathogenicity, tissue tropism, and genomic features of the highly attenuated 
Fig. 6 Contribution of an expression cassette in the F14.5L locus on the attenuation of GLV-1h68. a The schematic presentation of the $F 14.5 \mathrm{~L}$-null and revertant viruses derived from GLV-1h68. b The replication of the $F 14.5 L$-null and revertant viruses was studied in CV-1 cells, and compared to that of GLV-1h68. The virulence of the $F 14.5 \mathrm{~L}$-null and revertant viruses was investigated in nude mice by an intranasal application of $2 \times 10^{6} \mathrm{pfu}$ of individual virus per mouse. The survival and body weight of the mice were monitored for 10 weeks and presented in $\mathbf{c}$ and $\mathbf{d}$ a

GLV-1h68

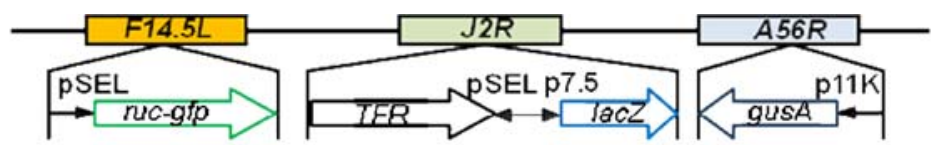

GLV-1h71

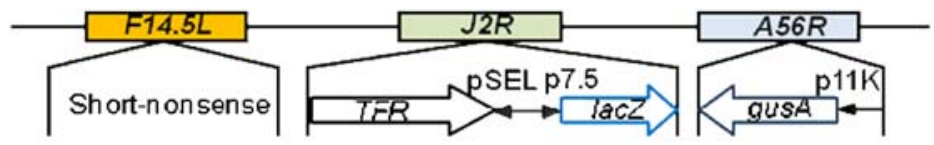

GLV-1e135

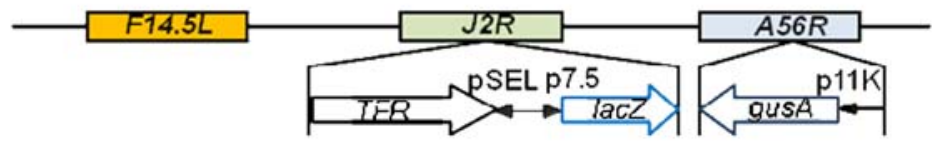

b

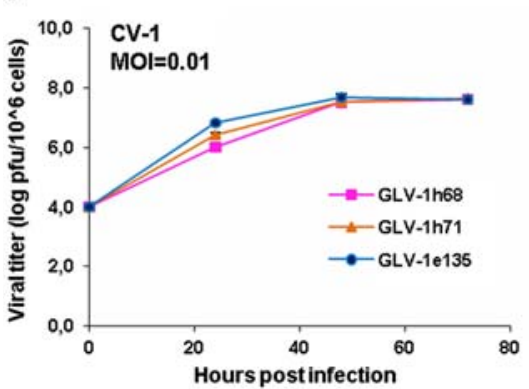

c
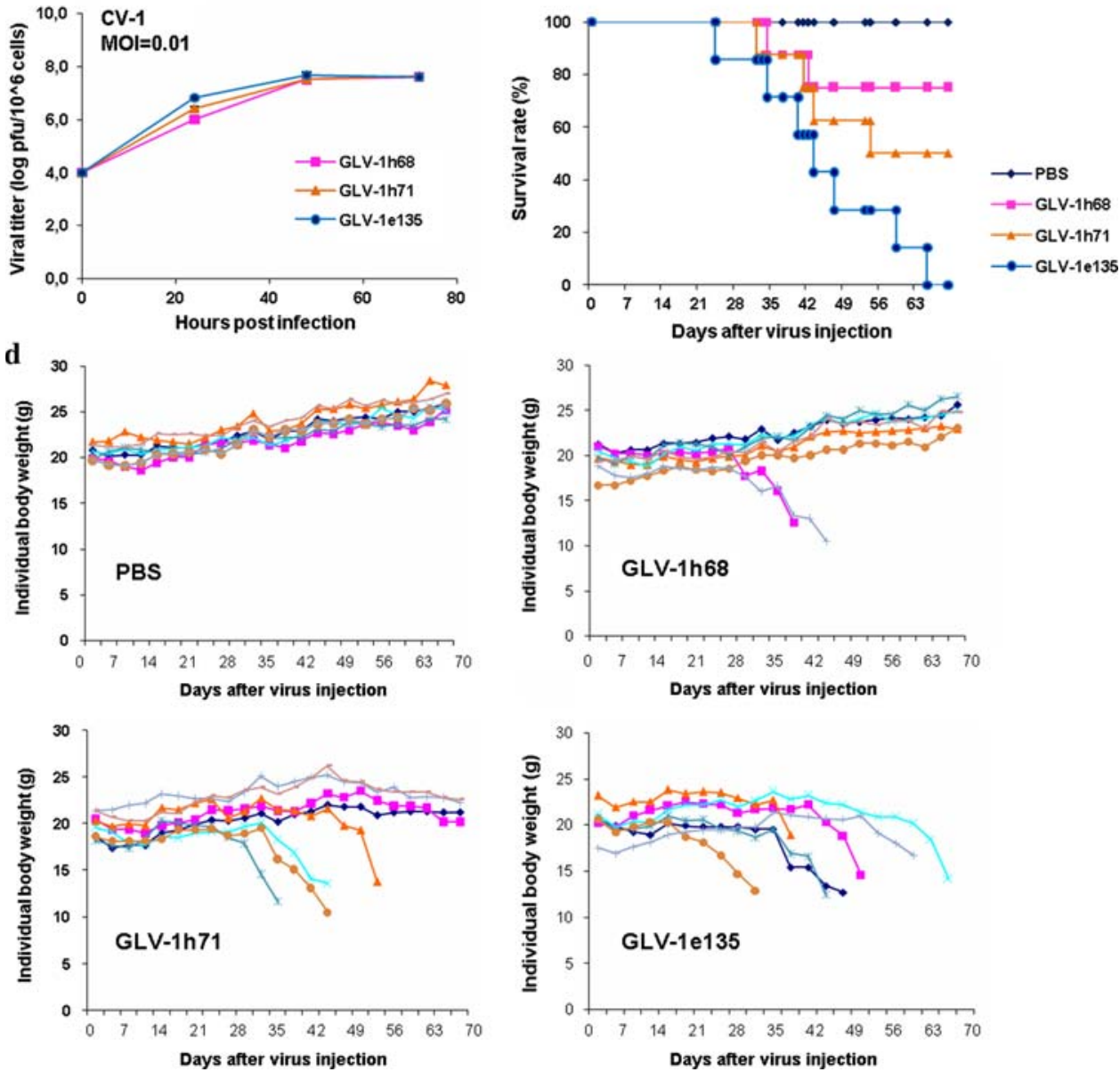

recombinant GLV-1h68 with WR, COP and related viridae. The in vivo studies show the reduced pathogenicity phenotype of GLV-1h68, previously confirmed in female nude mice bearing GI-101A breast tumor xenografts (Zhang et al. 2007), and now in male nude mice bearing C6 glioma and a melanoma model in immunocompetent mice. The comparative genome sequence analysis offered detailed functions of all infection-involved individual genes, and showed the uniqueness of GLV-1h68 and its evolutionary relationship. These findings provided a foundation for further studies on the effects of individual genes and gene variants. Detailed experimental examination of the effects of individual ORFs is time-consuming and will require a number of separate studies. Our study here focused on the effects of individual disruptions and inserted cassettes in the F14.5L, J2R and A56R loci of GLV-1h68 that resulted in reduced pathogenicity in mice. This is the first study attempting to differentiate the contribution of an inactivated viral gene (e.g. F14.5L) from that of an expression cassette (in the F14.5L locus) on the attenuation of a VACV (e.g. GLV-1h68). In the following discussion, we present the combined insights from both 
the bioinformatics and the experimental approaches applied in this study.

Comparison choice regarding different vaccinia strains

Some of the vvDD strains and the Wyeth $T K$-strains have been used as oncolytic strains under clinical investigation. They would be ideal candidates for comparison with GLV-1h68, which is now also as GL-ONC-1 undergoing a Phase I/II trial. Unfortunately, the genome sequence for any of these strains is not available or publicly accessible. The vvDD strains were derived from the WR strain, with the double deletion of VGF and TK. However, it is not safe to assume that a vvDD strain would have a similar genomic sequence as that of wt WR, given the fact that GLV-1h68, derived from an isolate from LIVP, showed a unique genetic make up very different from LIVP. Therefore, we chose to use the WR wt, which has a complete genome sequence for analyzing, and a WR $T K^{-}$strain, since we know that LIVP (wt) was a $T K^{-}$ strain, as the best available strains for comparison. On top of that, the WR strain has been the most widely studied strain in laboratories all over the world. We would gain more insight from the comparison with these laboratory WR strains.

Even though we did not directly compare our strains with the vvDD strains or the Wyeth $T K^{-}$strains, from published data, we can get a general idea what could be predicted from a direct comparison. During the construction of a vvDD strain, two copies of the viral VGF genes were first inactivated by the insertion of the lacZ gene under the control of the p11 promoter, and the resulting mutant vSC20 (Buller et al. 1988) was used as the parental virus for the double deletion mutant vVDD, in which the $T K$ gene was also inactivated by the insertion of one or more foreign gene expression cassettes. In one of the vvDD strains, vvDD-GFP (McCart et al. 2001), the EGFP gene under the control of a synthetic early-late promoter, and the gpt gene under the control of the p7.5 promoter were inserted in the TK locus. In nude mice injected with $10^{7}$ pfu of vvDDGFP, the median viral recovery from ovary tissues was as high as $8.6 \times 10^{6} \mathrm{pfu} / \mathrm{mg}$ total protein 8 days after the infection, suggesting that neither VGF nor TK was responsible for gonadotropism in female mice. However, both $\mathrm{VGF}^{-}$and $\mathrm{TK}^{-}$contributed to reduced viral replication in mouse brain. If we would like to find out which gene(s) contribute to gonadotropism in female mice, a genomic comparison with GLV-1h68 would help to narrow down the selection of the candidates. Of course, considerable amount of studies are required to pinpoint the exact gene(s). We hope that with this work including the detailed triple genome comparison, the combined efforts from our scientific community would speed up the discovery process, and eventually, an optimized oncolytic virus with maximum oncolytic activity yet minimum pathogenicity will become available to benefit cancer patients.

\section{ORFs involved in host-range selection}

Many differences in ORFs among the GLV-1h68, WR and COP strains can be attributed to the ORFs encoding ankyrin-like proteins (Table 1). Because these proteins control the interactions between the integral membrane proteins and the elements of the cell cytoskeleton, the proteins could be involved in functions related to transcriptional initiation, cell cycle regulation, cytoskeleton formation, ion transport and signal transduction (Lux et al. 1990; Mosavi et al. 2004). It has been proposed that different sets of the ankyrin-like proteins encoded by VACVs, as well as the level of their synthesis, allow for the fine regulation of virus replication and the determination of tissue tropism in infected animals (Shchelkunov et al. 1993). Recent studies on the M-T5 host-range protein of myxoma virus, which contains multiple ankyrin repeats, illustrate that interactions of M-T5 with Akt and cullin-1 (both components of host cell signaling networks) can have a profound impact on poxvirus tropism (Johnston et al. 2005; Wang et al. 2006).

It has been described that the deletion of two vaccinia host-range genes $(K 1 L$ and $C 7 L)$ greatly reduces the ability of VACV to replicate in a variety of human cell lines (Gillard et al. 1986). Restoration of the K1L gene in the MVA genome, however, failed to release the virus from its host restriction on monkey or human cells (Perkus et al. 1990), suggesting that there are other host-range determination genes yet to be recognized. $K 1 L$ and $C 7 L$ seemed to be well conserved among GLV-1h68, WR and COP. Yet, we found that, while GLV-1h68, along with the other two recombinant viruses, infected and replicated in human, monkey and rat cells, it did not replicate in mouse embryonic fibroblasts (MEFs) (Zhang et al. 2007; Fig. 5b). In contrast, wt-LIVP did replicate in MEFs, giving a virus yield of just 10-fold less than wt-WR (Fig. 5b). It is reasonable to speculate that the restricted replication of these three recombinant viruses in MEFs was caused by the inactivation of the $F 14.5 \mathrm{~L}$ locus. Our unpublished data, however, suggest that the disrupted $F 14.5 L$ was not responsible for this restricted replication. We have observed that a virus isolate derived from GLV-1h68, which had a restored $F 14.5 L$, failed to replicate in the MEFs as well (data not shown). Now we know that wt-LIVP was a mixed population, and that GLV-1h68 was derived from a unique isolate from this mixed population, a genome sequencing and comparative analysis with an LIVP isolate capable of replicating in MEFs would help to identify genes responsible for the restricted replication of GLV-1h68 in primary mouse cells. 


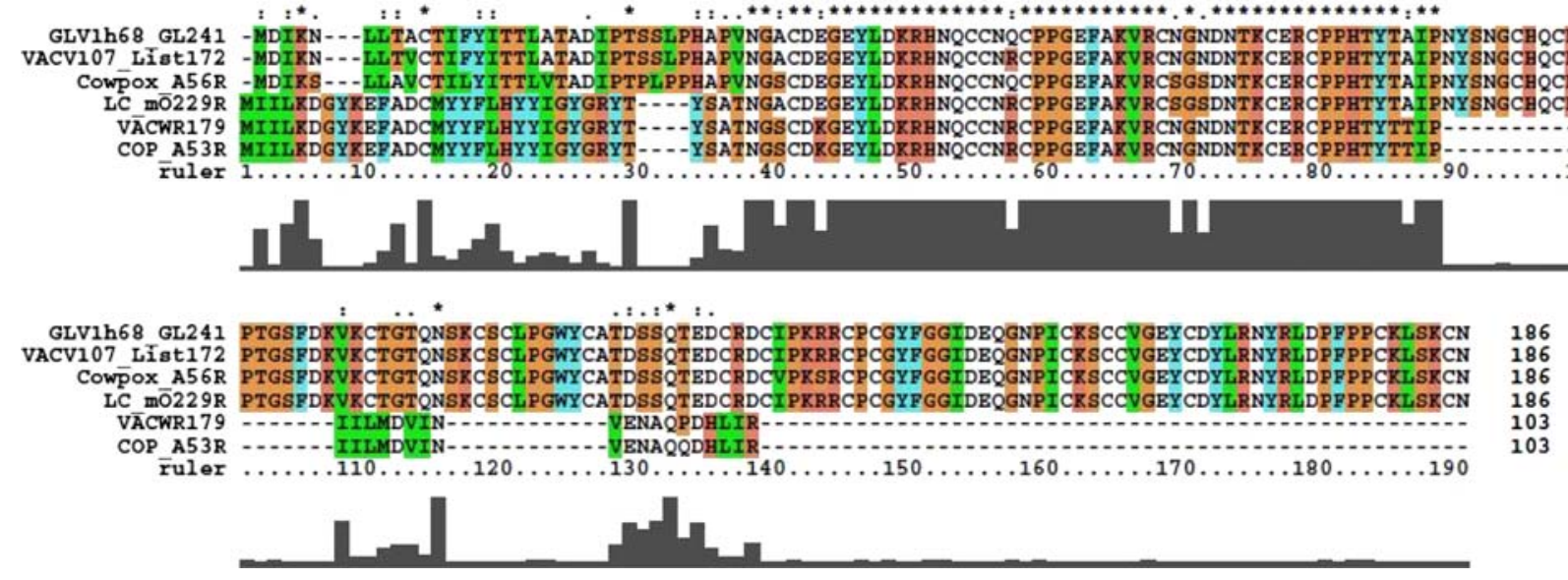

Fig. 7 Sequence alignment of $\operatorname{crm} C$ gene for selected poxvirus strains. The ORFs for $\mathrm{CrmC}$ in selected poxvirus strains were translated into amino acids and their sequences were aligned using ClustalX. GLV-1h68 exhibits an intact $\mathrm{crmC}$ gene (GL241), which is highly identical to List172 in Lister isolate VACV107, and A56 gene

ORFs involved in host immune modulation

$B 28 R$ and $A 53 R$, encoding two vTNFR proteins, are equivalent to Cowpox virus (CPV) genes cytokine response modifier B $(\mathrm{crmB})$ and $\mathrm{crm} C$, respectively. However, both $B 28 R$ and $A 53 R$ are fragmented and found to be inactive in most VACV strains, including WR and COP (Alcami et al. 1999). Nevertheless, GLV-1h68 was found to carry an intact A53R gene (GL241), which encoded a secreted TNFreceptor-like protein of 186aa that was the same length as CPV crmC (Fig. 7). CrmC was also found intact in the Lister isolate VACV107 (GenBank accession no. DQ121394). As a soluble vTNFR that interferes with host immune functions, $A 53 R$ in GLV-1h68 could be a potential candidate for disruption, in order to further attenuate the virus.

Unlike USSR, Evans and other Lister derivatives, GLV-1h68 did not have a crmE-like sequence in the genome. USSR recombinants lacking $c r m E$ were found to be attenuated, while the expression of an inserted $\mathrm{crmE}$ gene in the genome of WR resulted in enhanced virulence (Reading et al. 2002). The crmE deficiency in GLV-1d27 or GLV-1h68, therefore, may have contributed to a greater attenuation in comparison to wt-LIVP (the majority of wt-LIVP was crmE positive). VACV strains Lister, USSR and Evans also express the v-GAAP protein. The deletion of this gene from VACV does not affect virus replication in cell cultures, but significantly alters virus virulence in murine infection models (Gubser et al. 2007). GLV-1h68 does not have a v-GAAP gene. This gene would therefore be a good candidate to be inserted into the genome of GLV-1h68 to bring additional attenuation to the virus. in Cowpox virus, all of which contain a typical signal peptide sequence predicted by SignalP 3 software. Lister strain LC16mO seems to have a full length protein, but its $\mathrm{N}$-terminal sequence suggests that this protein is not secreted. The $\mathrm{crmC}$ genes in VACV strains Copenhagen and WR are truncated

It has been shown that deletion of the CPV $38 \mathrm{~K}$ gene encoding the well-known orthopoxvirus serpin (also known as SPI-2 or CrmA) in either the cowpox or rabbitpox virus genome resulted in attenuation in murine intranasal models (Thompson et al. 1993). The deletion mutants from the WR strain that lack the SPI-2 or SPI-1 coding sequence display similar in vitro replication profiles and in vivo virulence as wt-WR and the revertant viruses (Kettle et al. 1995). However, the derivative of the WR strain with both SPI- 1 and $S P I-2$ deleted has been shown to be significantly attenuated and less pathogenic to mice (Guo et al. 2005). The inconclusive findings on SPI-1 and SPI-2 may have resulted from the difference in the way the "deletion" mutants are constructed (more details in later discussion). Nevertheless, if inactivation of the SPI-2 gene indeed reduces virulence of the virus, the fragmented SPI-2 gene in GLV-1h68 (GL266 and GL267) may have contributed to its attenuation. Furthermore, the lack of a functional SPI-2 gene product may enhance the immunogenicity of the virus (Zhou et al. 1990).

It has been observed that A39R induces robust responses in human monocytes, including cell aggregation, induction of proinflammatory cytokines, and upregulation of the monocyte cell surface marker ICAM-1. All of these effects suggest that A39R influences the immune responses of vaccinia-infected hosts (Comeau et al. 1998). The A39R protein is found to be expressed by VACV strains COP, Evans, Lister, and USSR, but not WR. Expression of an inserted COP A39R gene in the WR strain was found to be associated with an increase in infiltration and oedema formation, suggesting that the A39R protein has pro-inflammatory properties (Gardner et al. 2001). The fact that $A 39 R$ was intact in LIVP and in GLV-1h68, but truncated in WR, 
suggests differences in immunogenicity, but requires further experimental confirmation.

\section{Genome evolution of GLV-1h68}

Our data also suggested a unique infection and replication profile of GLV-1h68 that was distinctively different from its parental strain LIVP. This distinction was particularly evident in mouse primary cells, which warranted a closer look at its genome evolution. A comparison of the sequence of the major PCR product on the right terminal region of wt-LIVP with GLV-1h68 supports our hypothesis that GLV-1h68 was a unique isolate that differed from the majority of the wt-LIVP population (Figs. 3, 4). GLV-1h68 showed a unique genetic makeup which shared genes similar to those of WR strains.

These changes illustrated that the parental strain of GLV-1h68 probably underwent two major rearrangement events. First, there was perhaps a genetic exchange with a WR-like VACV strain regarding the right terminal region. The C-terminal of GL273 was most likely the site of the recombination segment, since it was highly homologous to WR199. GL274, GL275 and GL276 were also acquired during the same genetic exchange, as they were similar to the downstream WR200, WR202 and WR201 genes, respectively. A second major rearrangement occurred when the parental strain lost part of its newly acquired WR-like sequences at the region, and a copy of its left terminal sequence, GLO01 to GLO15 genes, was duplicated, and then appeared in its right terminal region. This exchange probably occurred in a laboratory where WR strains and the parental strains of GLV-1h68 were used at the same time. The sequence of the major PCR product on the right terminal region of GLV-1d27 or GLV-1f65 was not determined, but it is very likely to be similar, if not identical, to that of GLV-1h68, given the fact that the same size PCR product was obtained, and the history of stepwise virus construction and purification.

The contribution of $F 14.5 \mathrm{~L}$ inactivation

F14.5L, reported as WR053.5 in WR, encodes a protein of 49 amino acids with a secretory signal peptide. This ORF was very well conserved across different VACV strains, as well as in other poxviruses. There is a NotI restriction site in the middle of $F 14.5 \mathrm{~L}$ sequence, which was used to insert a $R U C-G F P$ expression cassette to create the recombinant virus GLV-1d27, and subsequently the double-mutant virus GLV-1f65, and the triple-mutant GLV-1h68 (Timiryasova et al. 2000; Zhang et al. 2007). Our studies with the $F 14.5 \mathrm{~L}$-revertant and the $F 14.5 \mathrm{~L}$-null viruses suggest that the $F 14.5 \mathrm{~L}$ locus in viral genome can be disrupted as a way to attenuate VACV. The effect of inactivation of $F 14.5 \mathrm{~L}$ locus by itself was not very substantial, but it would likely become statistically significant with a larger sample size. The expression of an exogenous gene in the $F 14.5 \mathrm{~L}$ locus puts extra transcriptional and translational burden on the virus and, together with the inactivation of $F 14.5 \mathrm{~L}$, resulted in a significantly attenuated virus: GLV-1h68. It is possible that the expressed GFP could be toxic to some host cells that would inhibit viral replication. However, our unpublished data suggest that other foreign genes expressed in the same locus also attenuated the virus to similar extent; therefore the reduced virulence was not likely due to the toxicity brought by GFP. In a recent publication, WR53.5 is found to be an envelope protein of an intracellular mature virus that mediates cell adhesion (Izmailyan and Chang 2008). In that study, the WR53.5-deletion mutant virus, which contains a gpt expression cassette in the WR53.5 locus, exhibits reduced virulence in mice. It is conceivable that the expression of $g p t$, in addition to the inactivation of WR53.5L, may have contributed to the reduced virulence.

\section{The contribution of $J 2 R$ inactivation}

The TK negative phenotype was confirmed for the wild type LIVP population. A TK ${ }^{-}$virus requires TTP for DNA synthesis, leading to a preferential viral replication in dividing cells and ultimately enhanced tumor specificity and minimal infection of neighbor tissues. The $\mathrm{TK}^{-}$phenotype may explain, at least in part, why the wt-LIVP strain appeared to be less virulent than wt-WR in animal studies (Fig. 5c; Table 2); however, it cannot explain the observed differences in neurovirulence and gonadotropism between LIVP and WR strains. We saw earlier in female nude tumor-bearing mice that, even as disruption of the $T K$ gene in the WR genome contributed significantly to reduced viral entry or replication in brain tissues (a 3-log reduction), merely a 2.5 -fold reduction was observed in ovary tissues (Zhang et al. 2007). In this study, the disruption of the $T K$ gene in the WR genome resulted in on average a 7-fold and a 58-fold reduction in viral titers in the brain and testis tissues, respectively (Table 2). We therefore conclude that, while a $\mathrm{TK}^{-}$phenotype in the WR strain significantly reduced its neurovirulence (more evident in female mice) and reduced gonadotropism in male mice, it had little effect on gonadotropism in female mice. In contrast, despite the fact that the brain and ovary tissue were found to be free of viral particles in mice treated with either wt-LIVP or GLV1 h68 (both were $\mathrm{TK}^{-}$) (Zhang et al. 2007), the wt-LIVP did show a similar viral titer in testes as the WR strains in this new study (Table 2). We suspect that gene(s) other than the $\mathrm{TK}^{-}$phenotype contributed to the absence of gonadotropism in mice observed for the three recombinant viruses derived from LIVP, including GLV-1h68. However, it was not clear how much a $\mathrm{TK}^{-}$phenotype in the LIVP strains 
might have contributed to the absence or reduced neurovirulence and gonadotropism in mice.

It was also shown that the distribution of GLV-1f65 was further reduced in the normal tissues when compared to those injected with GLV-1d27 (Table 2). Both viruses were $\mathrm{TK}^{-}$, and the only difference was that GLV-1f65 contained a foreign gene expression cassette in its $J 2 R$ locus. Similar to the foreign gene (RUC-GFP) expression in the $F 14.5 \mathrm{~L}$ locus that contributed to the attenuation of GLV-1h68, the expression of lac $Z$ in the $J 2 R$ locus may have further contributed to the attenuation of GLV-1f65, and the attenuation of GLV-1h68. In the survival study reported previously (Zhang et al. 2007) the mice injected with GLV-1f65 did outlive the ones injected with GLV-1d27.

\section{The contribution of $A 56 R$ inactivation}

The A56R gene, which encodes hemagglutinin, is another nonessential gene that can be inactivated to obtain a more attenuated virus. It has been observed that inactivation of the $H A$ gene leads to greater attenuation of WR than of LC16mO (a temperature-sensitive and lower neurovirulence strain isolated from the Lister LO strain) and to no apparent attenuation of LO-1 (an isolate of Lister Elstree strain) (Shida et al. 1988). In our studies, disruption of the $H A$ gene by the insertion of an expression cassette further attenuated the virus (Zhang et al. 2007). While mice injected with GLV-1f65 ( $F 14.5 L^{-}$and $\left.T K^{-}\right)$lived up to 116 days after virus injection, all of the mice injected with GLV-1h68 (F14.5L $L^{-}, T K^{-}$and $\left.H A^{-}\right)$survived for the duration of study (130 days post virus infection). Further studies are needed to investigate how much of the attenuation in GLV-1h68 could be attributed to the inactivation of $H A$ itself, and how much was contributed by the expression of a foreign gene (gusA) in the A56R locus.

\section{The contribution of foreign gene expression}

GFP expression could be toxic to the host cells and may have reduced the replication of GLV-1h68, thus the GFP would be the cause of the reduced pathogenicity. Data published before (Zhang et al. 2007) and here suggest that the expression of $\beta$-galactosidase in the TK locus attenuated the virus as well. We are currently conducting a whole set of studies in which the strength of different viral promoters, the size of the transgenes, the 2nd structure of the transcripts, and the locus of the insertions are the factors under investigation. Our preliminary data suggest that the observation that transgene expression appears to reduce viral replication is generally true. All these data, when they become available, will be presented in a new study.

A review of published data so far by other investigators also suggests that the expression of inserted foreign gene(s) may play a role in reduced virulence observed with those engineered viruses. For example, when the SPI-1 or SPI-2 gene was simply deleted, no reduced virulence was observed (Kettle et al. 1995). But when both SPI-1 and SPI-2 genes were inactivated by the insertion of a functional gpt expression cassette and an Escherichia coli lacZ gene expression cassette, respectively, the resulting mutant was markedly attenuated (Guo et al. 2005). It is possible that the effect of individual gene deletion was not significant, but the inactivation of both genes made the attenuation more evident. The way the double-mutant virus was constructed, however, has made us wonder how much of the attenuation was actually contributed by the expression of transgenes.

\section{General considerations and conclusions}

We investigated the genetic differences between GLV-1h68 and related VACVs. A first comprehensive analysis including function predictions for all found genome differences is easily gained by bioinformatics and comparative genome analysis, even though this method cannot detect and rule out further differences on smaller polymorphisms (e.g. SNPs). Such more subtle effects and differences require extensive genetic and molecular studies to test individual genes in a number of strains and their direct effects in different infection scenarios. Table 3 summarizes a number of genome differences, as examined and discussed here. It is important to realize that the functions of viral genes could be strain-specific, tissue-specific, or host-specific. The virulence genes, which are important for one VACV strain, may play a minimal role in the virulence for another VACV strain. The inactivation of a certain viral gene may influence the replication of VACV in one tissue type but not other tissues in the same host, or may reduce the pathogenicity of the virus to one host species but not to others. There might also be synergistic effects involving multiple viral genes. In this report, we focused our analysis on the differences between the viral genes that were absent or fragmented in certain strains and the corresponding viral genes in the Lister, WR and COP strains. Using sequences available from additional VACV genomes, in combination with functional transcription and proteome analysis, it is possible to rationally design more optimized oncolytic virus strains to benefit cancer therapy in human patients. The analysis of GLV-1h68 is an important step towards this goal, as demonstrated here by direct sequence data, functional tests and tissue tropism.

This study also suggests that insertion of a foreign gene expression cassette in a nonessential locus in the viral genome is a practical way to attenuate VACVs, especially if the nonessential locus itself contains a virulence gene. The challenge will then be to reduce the 
Table 3 Significant specific differences

\begin{tabular}{|c|c|}
\hline \multirow[t]{4}{*}{ 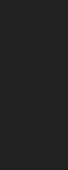 } & Triple comparison \\
\hline & TNF-alpha-receptor-like protein (WRO02, 217) \\
\hline & Ankyrin-like protein (WR006, 213; C21L, B27R) \\
\hline & Ankyrin-like protein (WR007, 212; C20L, B26R) \\
\hline \multirow[b]{2}{*}{ ᄋి } & Ankyrin-like protein (WR008, 211; C19L, B25R) \\
\hline & Ankyrin-like protein $(G L 005,287)$ \\
\hline \multirow{2}{*}{$\stackrel{0}{3}$} & Putative host-range protein (GLO06, 286; C18L, B24R) \\
\hline & Putative host-range polypeptide ( $C 17 L, B 23 R$; fragment: $G L 007+008,284+285$ ) \\
\hline \multirow{4}{*}{ 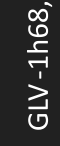 } & SPI-1 (GLOO9, 283; WR205; C12L) \\
\hline & Secreted epidermal growth factor (GL010, 282; WR009, 210; C11R) \\
\hline & Fragment IL-1 receptor antagonist (GL011, 280; WR010, 209; C10L) \\
\hline & IL-18-binding protein (GL015, 277; WRO13) \\
\hline \multirow{7}{*}{ 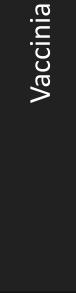 } & Ankyrin-like protein (WR014; fragment: GL016+017) \\
\hline & Ankyrin-like protein (GL018; WRO15) \\
\hline & Ankyrin-like protein (GL019; fragment: WR016+017) \\
\hline & Semaphorin-like protein (GL221; A39R; fragment: WR163+164) \\
\hline & TNF receptor (GL241; fragment: WR179; A53R) \\
\hline & SPI-2/CrmA (WR195; fragment: GL266+267; B13R+B14R) \\
\hline & IL-1-beta-receptor (GL270; WR197; fragment: $270+B 16 R$ ) \\
\hline \multirow{10}{*}{$\begin{array}{l}\infty \\
\stackrel{0}{0} \\
\frac{1}{7} \\
\frac{1}{3} \\
0\end{array}$} & Modification in GLV-1h68 \\
\hline & Unknown protein (GL068; WR053.5; F14.5; disrupted in GLV-1h68) \\
\hline & Thymidine kinase (WR094; J2R; fragment: GL120+121, disrupted in GLV-1h68) \\
\hline & Hemagglutinin (GL245; WR181; A56R; disrupted in GLV-1h68) \\
\hline & Specific for Lister \\
\hline & Island (259R-262L-RTR01R) specific for Lister \\
\hline & -CrmE (259R, absent from GLV-1h68, WR, COP) \\
\hline & -Hypothetical protein genes specific for Lister $(260 R, 261 R, 262 L$ and $R T R 01 R)$ \\
\hline & Absent from Lister, present in GLV -1h68 and WR \\
\hline & Island $(G L 274,275,276)$ specific for GLV $-1 \mathrm{~h} 68$ and WR \\
\hline \multirow{2}{*}{ 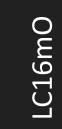 } & $\begin{array}{l}\text {-IFN-alpha/beta-receptor secreted protein (GL274; WR200, absent from other Lister } \\
\text { strains) }\end{array}$ \\
\hline & -Hypothetical Protein genes (GL275, 276; WR201, 202) \\
\hline \multirow{11}{*}{ 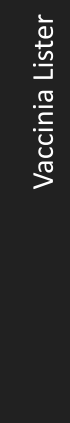 } & Copy number diversity \\
\hline & Island (GL277-283, GL009-015) has 2 copies in GLV-1h68, but only 1 in Lister (004L-009L) \\
\hline & -Serine protease inhibitor-like SPI-1 (004L; GL009, 283) \\
\hline & -Secreted epidermal growth factor (005R; GL010, 282) \\
\hline & -Fragment IL-1 receptor antagonist (007L; GL011, 280) \\
\hline & -Zinc finger-like protein (008R; fragment : GL013+014, GL0278+279) \\
\hline & -IL-18-binding protein (009L; GL015, 277) \\
\hline & Other changes \\
\hline & Thymidine kinase (112R; fragment: GL120+121) \\
\hline & Host-range polypeptide (LTRO2L, RTR02R; fragment: GL007+008, GL284+285) \\
\hline & Ankyrin-like protein (258R:413aa extends into GL273:574aa with different end-terminus) \\
\hline
\end{tabular}

virulence of the virus without compromising too much on the replication competency of the virus, the key to its oncolytic activity.

Acknowledgments This work was supported by research and development grants from Genelux Corporation and in part by Deutsche Forschungsgemeinschaft (DFG) TR34/A5 and Bundesministerium für Bildung und Forschung (BMBF) Pathogenomik (CL) and Land Bavaria (TD). The authors wish to thank Dr. Tatyana Timiryasova for her scientific and technical contribution, Mr. Terry Trevino and Ms. Melody Jing for their technical support, and Ms. Andrea Feathers for editorial support.
Open Access This article is distributed under the terms of the Creative Commons Attribution Noncommercial License which permits any noncommercial use, distribution, and reproduction in any medium, provided the original author(s) and source are credited.

\section{References}

Al'tshtein AD, Zakharova LG, Loparev VN, Pashvykina GV, Gorodetskii SI (1985) Isolation of a recombinant vaccinia virus based on the LIVP strain inducing the surface antigen of the hepatitis B virus. Dokl Akad Nauk SSSR 285:696-699 
Alcami A, Khanna A, Paul NL, Smith GL (1999) Vaccinia virus strains Lister, USSR and Evans express soluble and cell-surface tumour necrosis factor receptors. J Gen Virol 80:949-959

Antoine G, Scheiflinger F, Dorner F, Falkner FG (1998) The complete genomic sequence of the modified vaccinia Ankara strain: comparison with other orthopoxviruses. Virology 244:365-396

Bateman A, Coin L, Durbin R, Finn RD, Hollich V, Griffiths-Jones S, Khanna A, Marshall M, Moxon S et al (2004) The Pfam protein families database. Nucl Acid Res 32:D138-D141

Besemer J, Borodovsky M (2005) GeneMark: web software for gene finding in prokaryotes, eukaryotes and viruses. Nucl Acid Res 33:W451-W454

Buller RM, Smith GL, Cremer K, Notkins AL, Moss B (1985) Decreased virulence of recombinant vaccinia virus expression vectors is associated with a thymidine kinase-negative phenotype. Nature 317:813-815

Buller RM, Chakrabarti S, Cooper JA, Twardzik DR, Moss B (1988) Deletion of the vaccinia virus growth factor gene reduces virus virulence. J Virol 62:866-874

Comeau MR, Johnson R, DuBose RF, Petersen M, Gearing P, VandenBos T, Park L, Farrah T, Buller RM et al (1998) A poxvirus-encoded semaphorin induces cytokine production from monocytes and binds to a novel cellular semaphorin receptor, VESPR. Immunity 8:473-482

Delcher AL, Bratke KA, Powers EC, Salzberg SL (2007) Identifying bacterial genes and endosymbiont DNA with Glimmer. Bioinformatics 23:673-679

Earl PL, Moss B, Wyatt LS, Carroll MW (1998) Generation of recombinant vaccinia viruses. In: Ausubel F, Brent R, Kingston R, Moore D, Seidman J, Smith J, Struhl K (eds) Current protocols in molecular biology, vol 3. Wiley, New York, pp 16.17.1-16.19.7

Falkner FG, Moss B (1990) Transient dominant selection of recombinant vaccinia viruses. J Virol 64:3108-3111

Gardner JD, Tscharke DC, Reading PC, Smith GL (2001) Vaccinia virus semaphorin A39R is a $50-55 \mathrm{kDa}$ secreted glycoprotein that affects the outcome of infection in a murine intradermal model. J Gen Virol 82:2083-2093

Gillard S, Spehner D, Drillien R, Kirn A (1986) Localization and sequence of a vaccinia virus gene required for multiplication in human cells. Proc Natl Acad Sci USA 83:5573-5577

Goebel SJ, Johnson GP, Perkus ME, Davis SW, Winslow JP, Paoletti E (1990) The complete DNA sequence of vaccinia virus. Virology 179:247-266, 517-563

Gubser C, Bergmaschi D, Hollinshead M, Lu X, van Kuppeveld FJM, Smith GL (2007) A new inhibitor of apoptosis from vaccinia virus and eukaryotes. PLoS Pathog 3:e17

Guo ZS, Naik A, O’Malley ME, Popovic P, Demarco R, Hu Y, Yin X, Yang S, Zeh HJ et al (2005) The enhanced tumor selectivity of an oncolytic vaccinia lacking the host range and antiapoptosis genes SPI-1 and SPI-2. Cancer Res 65:9991-9998

Henderson DA, Moss B (1999) Smallpox and vaccinia. In: Plotkin S, Orenstein W (eds) Vaccines. W.B. Saunders, Philadelphia, pp 74-97

Horton RM, Ho SN, Pullen JK, Hunt HD, Cai Z, Pease LR (1993) Gene splicing by overlap extension. Methods Enzymol 217:270279

Huson DH, Bryant D (2006) Application of phylogenetic networks in evolutionary studies. Mol Biol Evol 23:254-267

Izmailyan R, Chang W (2008) Vaccinia virus WR53.5/F14.5L protein is a new component of intracellular mature virus and is important for calcium-independent cell adhesion and vaccinia virus virulence in mice. J Virol 82:10079-10087

Johnston JB, Wang G, Barrett JW, Nazarian SH, Colwill K, Moran M, McFadden G (2005) Myxoma virus M-T5 protects infected cells from the stress of cell cycle arrest through its interaction with host cell cullin-1. J Virol 79:10750-10763
Joklik WK (1962) The purification of four strains of poxvirus. Virology 18:9-18

Katoh K, Kuma K, Toh H, Miyata T (2005) MAFFT version 5: improvement in accuracy of multiple sequence alignment. Nucl Acids Res 33:511-518

Kettle S, Blake NW, Law KM, Smith GL (1995) Vaccinia virus serpins B13R (SPI-2) and B22R (SPI-1) encode M(r) 38.5 and $40 \mathrm{~K}$, intracellular polypeptides that do not affect virus virulence in a murine intranasal model. Virology 206:136-147

Li G, Chen N, Feng Z, Buller RML, Osborne J, Harms T, Damon I, Upton C, Esteban DJ (2006) Genomic sequence and analysis of a vaccinia virus isolate from a patient with a smallpox vacciniarelated complication. Virol J 3:88

Liang C, Dandekar T (2006) InGeno-an integrated genome and ortholog viewer for improved genome to genome comparisons. BMC Bioinform 7:461

Lux SE, John KM, Bennett V (1990) Analysis of cDNA for human erythrocyte ankyrin indicates a repeated structure with homology to tissue-differentiation and cell-cycle control proteins. Nature 344:36-42

Mackett M, Smith GL, Moss B (1982) Vaccinia virus: a selectable eukaryotic cloning and expression vector. Proc Natl Acad Sci USA 79:7415-7419

McCart JA, Ward JM, Lee J, Hu Y, Alexander HR, Libutti SK, Moss B, Bartlett DL (2001) Systemic cancer therapy with a tumorselective vaccinia virus mutant lacking thymidine kinase and vaccinia growth factor genes. Cancer Res 61:8751-8757

Mosavi LK, Cammett TJ, Desrosiers DC, Peng ZY (2004) The ankyrin repeat as molecular architecture for protein recognition. Protein Sci 13:1435-1448

Perkus ME, Goebel SJ, Davis SW, Johnson GP, Limbach K, Norton EK, Paoletti E (1990) Vaccinia virus host range genes. Virology 179:276-286

Perrière G, Gouy M (1996) WWW-query: an on-line retrieval system for biological sequence banks. Biochimie 78:364-369

Reading PC, Khanna A, Smith GL (2002) Vaccinia virus CrmE encodes a soluble and cell surface tumor necrosis factor receptor that contributes to virus virulence. Virology 292:285-298

Shchelkunov SN, Blinov VM, Sandakhchiev LS (1993) Ankyrin-like proteins of variola and vaccinia viruses. FEBS Lett 319:163-165

Shen Y, Nemunaitis J (2005) Fighting cancer with vaccinia virus: teaching new tricks to an old dog. Mol Ther 11:180-195

Shida H, Hinuma Y, Hatanaka M, Morita M, Kidokoro M, Suzuki K, Maruyama T, Takahashi-Nishimaki F, Sugimoto M et al (1988) Effects and virulences of recombinant vaccinia viruses derived from attenuated strains that express the human T-cell leukemia virus type I envelope gene. J Virol 62:4474-4480

Sigrist CJ, Cerutti L, Hulo N, Gattiker A, Falquet L, Pagni M, Bairoch A, Bucher P (2002) PROSITE: a documented database using patterns and profiles as motif descriptors. Brief Bioinform 3:265-274

Smith TF, Waterman MS (1981) Identification of common molecular subsequences. J Mol Biol 147:195-197

Sonnhammer EL, Durbin R (1995) A dot-matrix program with dynamic threshold control suited for genomic DNA and protein sequence analysis. Gene 167:GC1-GC10

Stajich JE, Block D, Boulez K, Brenner SE, Chervitz SA, Dagdigian C, Fuellen G, Gilbert JGR, Korf I et al (2002) The Bioperl toolkit: Perl modules for the life sciences. Genome Res 12:1611-1618

Thompson JP, Turner PC, Ali AN, Crenshaw BC, Moyer RW (1993) The effects of serpin gene mutations on the distinctive pathobiology of cowpox and rabbitpox virus following intranasal inoculation of Balb/c mice. Virology 197:328-338

Thompson JD, Higgins DG, Gibson TJ (1994) CLUSTAL W: improving the sensitivity of progressive multiple sequence alignment through sequence weighting, position-specific gap penalties and weight matrix choice. Nucl Acids Res 22:4673-4680 
Thorne SH, Bartlett DL, Kirn DH (2005) The use of oncolytic vaccinia viruses in the treatment of cancer: a new role for an old ally? Curr Gene Ther 5:429-443

Timiryasova TM, Yu YA, Shabahang S, Fodor I, Szalay AA (2000) Visualization of vaccinia virus infection using the renilla-luciferase-GFP fusion protein. In: Case J, Herring P, Robison B, Haddock S, Kricka L, Stanley P (eds) Proceeding of the 11th international symposium on bioluminescence \& chemiluminescence. World Scientific Publishing Co. Pt. Ltd. River Edge (NJ), pp 457-460

Upton C, Slack S, Hunter AL, Ehlers A, Roper RL (2003) Poxvirus orthologous clusters: toward defining the minimum essential poxvirus genome. J Virol 77:7590-7600

Verardi PH, Jones LA, Aziz FH, Ahmad S, Yilma TD (2001) Vaccinia virus vectors with an inactivated gamma interferon receptor homolog gene (B8R) are attenuated in vivo without a concomitant reduction in immunogenicity. J Virol 75:11-18
Wang G, Barrett JW, Stanford M, Werden SJ, Johnston JB, Gao X, Sun M, Cheng JQ, McFadden G (2006) Infection of human cancer cells with myxoma virus requires Akt activation via interaction with a viral ankyrin-repeat host range factor. Proc Natl Acad Sci USA 103:4640-4645

Yu YA, Shabahang S, Timiryasova TM, Zhang Q, Beltz R, Gentschev I, Goebel W, Szalay AA (2004) Visualization of tumors and metastases in live animals with bacteria and vaccinia virus encoding light-emitting proteins. Nat Biotechnol 22:313-320

Zhang Q, Yu YA, Wang E, Chen N, Danner RL, Munson PJ, Marincola FM, Szalay AA (2007) Eradication of solid human breast tumors in nude mice with an intravenously injected light-emitting oncolytic vaccinia virus. Cancer Res 67:10038-10046

Zhou J, Crawford L, McLean L, Sun XY, Stanley M, Almond N, Smith GL (1990) Increased antibody responses to human papillomavirus type $16 \mathrm{~L} 1$ protein expressed by recombinant vaccinia virus lacking serine protease inhibitor genes. J Gen Virol 71:2185-2190 